\title{
HAUTEUR ASYMPTOTIQUE DES POINTS DE HEEGNER
}

\author{
GUILLAUME RICOTTA - THOMAS VIDICK
}

\begin{abstract}
The asymptotic behaviour of the Néron-Tate height of Heegner points on a rational elliptic curve attached to an arithmetically normalized new cusp form $f$ of weight 2, level $N$ and trivial character is studied in this paper. By Gross-Zagier formula, this height is related to the special value at the critical point for the derivative of the Rankin-Selberg convolution of $f$ with a certain weight one theta series attached to some ideal class of some imaginary quadratic field. Asymptotic formula for the first moments asociated to these Dirichlet series are proved and experimental results are carefully discussed.
\end{abstract}

En l'honneur du Professeur Henryk Iwaniec, pour l'ensemble de son oeuvre analytique et pour son titre de Docteur Honoris Causa de l'Université de Bordeaux 1.

TABLE DES MATIÈRES

\begin{tabular}{|c|}
\hline 1. Description de la problématique \\
\hline 2. Préliminaires \\
\hline 3. Les traces \\
\hline 3.1. Mise en place \\
\hline 3.2. Courbes de rang analvtiaue 0 \\
\hline 3.3. Courbes de rang analvtioue 1 \\
\hline 4. Estimation asvmptotique de la hauteur des points de Heegnen \\
\hline 5. Analyse des résultats théoriques et numériques \\
\hline 5.1. Quelaues valeurs numériaues \\
\hline 5.1.1. Valeurs numériques de $C_{\operatorname{Tr}}$ et de $C_{\mathrm{P}}$ \\
\hline 5.1.2. Étude plus fine du rapport $C_{\mathrm{P}} / C_{\mathrm{T}}^{(0)}$ \\
\hline 5.2. Résultats expérimentaux \\
\hline 5.2.1. Comparaison entre les valeurs expérimentale et théorique de $C_{\mathrm{P}}$ \\
\hline 5.2.2. Étude plus fine des courbes $37 \mathrm{~A}$ et $37 \mathrm{~B}$ \\
\hline Références \\
\hline
\end{tabular}

Date: Version of 2 avril 2018.

2005 Mathematics Subject Classification: 11G50, 11M41. 


\section{Description de la problématique}

L'étude calculatoire systématique de la hauteur de Néron-Tate des points de Heegner sur différentes courbes elliptiques montre de grandes disparités. Si l'on considère deux courbes elliptiques de même conducteur $N$ alors les points de Heegner sur ces deux courbes sont l'image des mêmes points spéciaux de la courbe modulaire de niveau $N$ notée $\mathrm{X}_{0}(N)$ par la paramétrisation modulaire et on s'attend donc à ce que leurs hauteurs soient dans le même rapport que les degrés de ces paramétrisations. Cependant, la figure 1 montre que le comportement des hauteurs est très irrégulier : même si les courbes $37 \mathrm{~A}$ et 37B (dans la notation de Cremona $([\mathrm{Cr}])$ ) ont le même conducteur et le même degré, les points paraissent légèrement plus gros sur la 37B que sur la $37 \mathrm{~A}$.

Nous allons montrer que l'intuition se vérifie asymptotiquement : à conducteur fixé ${ }^{1}$, la moyenne sur une certaine sous-classe de discriminants de la hauteur des points de Heegner est proportionnelle au degré.

Pour cela, nous allons procéder à partir de la formule de Gross-Zagier ( raisonner sur les séries de Dirichlet et les fonctions $L$ en nous servant principalement d'un résultat de H. Iwaniec ([Iw] $)$.

Remerciements. Les auteurs remercient chaleureusement H. Darmon pour leur avoir suggéré cette problématique et pour ses nombreux conseils et encouragements. Ce travail a été réalisé à l'occasion d'un stage d'études à McGill University (Montréal) pour le second auteur et d'un stage post-doctoral à l'Université de Montréal (Montréal) pour le premier auteur. Les excellentes conditions de travail offertes par ces deux institutions ont fortement contribué à la réalisation de cet article. Le premier auteur a largement profité de la générosité et des conseils Mathématiques avisés de A. Granville lors de son stage post-doctoral.

\section{Préliminaires}

Soit $E$ une courbe elliptique définie sur $\mathbb{Q}$. Supposons pour simplifier que son conducteur $N$ est sans facteurs carrés et considérons l'ensemble

$$
\mathcal{D}:=\left\{d \in \mathbb{Z}_{-}^{*}, \mu^{2}(d)=1, d \equiv \nu^{2} \quad \bmod { }_{4} N,\left(\nu,{ }_{4} N\right)=1\right\}
$$

de discriminants. Pour $d$ dans $\mathcal{D}$, notons $\mathbb{H}_{d}$ le corps de classe de Hilbert du corps quadratique imaginaire $\mathbb{K}_{d}:=\mathbb{Q}(\sqrt{d})$ et souvenons-nous que $G_{d}:=\operatorname{Gal}\left(\mathbb{H}_{d} \mid \mathbb{K}_{d}\right)$ est isomorphe au groupe de classes de $\mathbb{K}_{d}$ de cardinal le nombre de classes $h_{d}$. Soit $X_{0}(N)$ la courbe modulaire de niveau $N$ classifiant les paires de courbes elliptiques $\left(E_{1}, E_{2}\right)$ reliées par une isogénie cyclique de degré $N$. Une description analytique sur $\mathbb{C}$ de cette courbe est donnée par le quotient du demi-plan de Poincaré complété $\mathbb{H} \cup(\mathbb{Q} \cup\{\infty\})$ par l'action par homographies du groupe de congruence $\Gamma_{0}(N)$.

\footnotetext{
${ }^{1}$ Toutefois, nous prendrons soin de garder explicite toute dépendance en le conducteur $N$ de la courbe.
} 


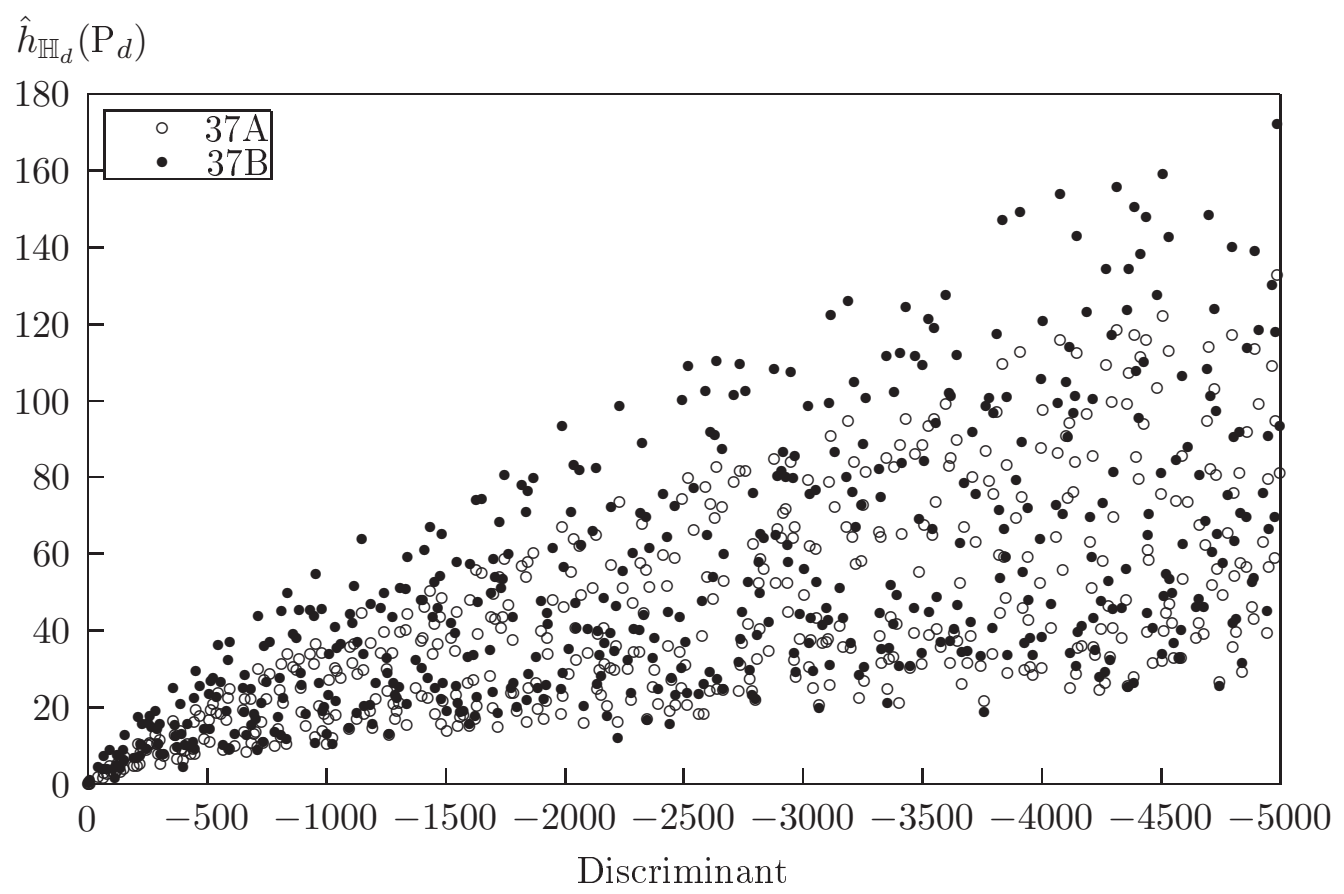

FIG. 1 - Comportement des points de Heegner sur les courbes 37A et 37B

Définition 2.1. Un point de Heegner de niveau $N$ et de discriminant $d$ est un couple ordonné $\left(E_{1}, E_{2}\right)$ de courbes elliptiques muni d'une isogénie cyclique de degré $N$ tel que $E_{1}$ et $E_{2}$ aient multiplication complexe par l'anneau des entiers $\mathcal{O}_{d}$ de $\mathbb{K}_{d}$.

Fixons une fois pour toute une racine carrée $s_{d}$ de $d$ modulo $4 N$ et désignons par $\mathfrak{n}_{d}$ l'idéal entier primitif de norme $N$ suivant :

$$
\mathfrak{n}_{d}:=\left(N, \frac{s_{d}+\sqrt{d}}{2}\right) .
$$

A $s_{d}$ fixé, l'ensemble des points de Heegner de niveau $N$ et de discriminant $d$ est en bijection avec le groupe de classes de $\mathbb{K}_{d}$ au sens suivant : si [a] est l'élément du groupe de classes de $\mathbb{K}_{d}$ associé à l'idéal entier primitif $\mathfrak{a}$ de $\mathcal{O}_{d}$ alors $\left(\mathbb{C} / \mathfrak{a}, \mathbb{C} / \mathfrak{a n}_{d}{ }^{-1}\right)$ est un point de Heegner de niveau $N$ et de discriminant $d$. Le point du demi-plan de Poincaré modulo $\Gamma_{0}(N)$ correspondant à ce point de Heegner est donné par $\frac{-B+\sqrt{d}}{2 A}$ modulo $\Gamma_{0}(N)$ où $(A, B, C)$ est la forme quadratique de discriminant $d$ correspondant à [a] et où $N \mid A$ et $B \equiv s_{d} \bmod 2 N$. 
Définition 2.2. Un point de Heegner de niveau $N$ et de discriminant d sur $E$ est l'image par la paramétrisation modulaire $\Phi_{N, E}: X_{0}(N) \rightarrow E$ d'un point de la forme

$$
\left(E_{1}, E_{2}\right)=\left(\mathbb{C} / \mathfrak{a}, \mathbb{C} / \mathfrak{a n}_{d}{ }^{-1}\right),
$$

où a est un idéal de l'anneau des entiers $\mathcal{O}_{d}$ de $\mathbb{K}_{d}$.

Notons $\mathrm{P}_{d}=\Phi_{N, E}\left(\left(E_{1}, E_{2}\right)\right)$, ce point ne dépend que de la classe de $\mathfrak{a}$ dans le groupe de classes de $\mathbb{K}_{d}$ (une fois $E$ et $d$ fixés). Notons également $\operatorname{Tr}_{d}=\operatorname{Tr}_{\mathbb{H}_{d} \mid \mathbb{K}_{d}}\left(\mathrm{P}_{d}\right)$. Les points de Heegner sont définis sur $E\left(\mathbb{H}_{d}\right)$ et sont permutés par $G_{d}([\mathrm{Gr}])$.

Si $R$ est un corps de nombres alors $\hat{h}_{R}(P)$ désigne la hauteur de Néron-Tate (comme définie dans [Si], VIII.9) prise sur $R$ du point $P$ à coordonnées dans $R$. On rappelle que si $S$ est une extension de degré fini de $R$ alors $\hat{h}_{S}(P)=[S: R] \hat{h}_{R}(P)$. Le but de cet article est d'étudier la valeur en moyenne, sur les $d$ dans $\mathcal{D}$ des deux objets suivants :

- $\hat{h}_{\mathbb{H}_{d}}\left(\mathrm{P}_{d}\right)$, hauteur de Néron-Tate sur $\mathbb{H}_{d}$ d'un quelconque des $h_{d}$ points de Heegner définis ci-dessus. Puisque $\hat{h}_{\mathbb{H}_{d}}$ est invariante sous l'action de $G_{d}$, cette hauteur est indépendante du point choisi,

- $\hat{h}_{\mathbb{K}_{d}}\left(\operatorname{Tr}_{d}\right)$, hauteur de Néron-Tate de la trace sur $\mathbb{K}_{d}$ d'un quelconque des points de Heegner $\mathrm{P}_{d}$ définis ci-dessus.

B.H. Gross et D. Zagier ( $\mathrm{GrZa}$ ) ont relié $\hat{h}_{\mathbb{H}_{d}}\left(\mathrm{P}_{d}\right)$ à la valeur de la dérivée en 1 de la série de Dirichlet obtenue en effectuant le produit de la fonction $L$ de Dirichlet associée au caractère primitif réel $\chi_{d}(m):=\left(\frac{d}{m}\right)$ de conducteur $|d|$ du corps $\mathbb{K}_{d}$ (le caractère de Kronecker du corps) par la convolution de Rankin-Selberg de $L(E \mid \mathbb{Q}, s)$ avec la fonction zeta $\sum_{n \geqslant 1} r_{d}(n) n^{-s}$ de la classe des idéaux principaux de $\mathbb{K}_{d}$ c'est-à-dire

$$
L_{d}(E, s):=\left(\sum_{\substack{m \geqslant 1 \\(m, N)=1}} \frac{\chi_{d}(m)}{m^{2 s-1}}\right) \times\left(\sum_{n \geqslant 1} \frac{a_{n} r_{d}(n)}{n^{s}}\right)
$$

où pour tout entier naturel non-nul $n, r_{d}(n)$ désigne le nombre d'idéaux principaux de $\mathbb{K}_{d}$ de norme $n$.

Théorème 2.1 (B.H. Gross-D. Zagier (1986)). Si E est une courbe elliptique définie sur $\mathbb{Q}$ et $d$ est dans $\mathcal{D}$ alors

$$
\begin{aligned}
L_{d}^{\prime}(E, 1) & =\frac{2 \Omega_{E, N}}{u^{2} \sqrt{-d}} \hat{h}_{\mathbb{H}_{d}}\left(\mathrm{P}_{d}\right), \\
L^{\prime}\left(E \mid \mathbb{K}_{d}, 1\right) & =\frac{2 \Omega_{E, N}}{u^{2} \sqrt{-d}} \hat{h}_{\mathbb{K}_{d}}\left(\operatorname{Tr}_{d}\right)
\end{aligned}
$$

où $L\left(E \mid \mathbb{K}_{d}, s\right)$ est la fonction $L$ de $E$ sur le corps $\mathbb{K}_{d}$, $2 u$ est le nombre de racines de l'unité de $\mathbb{K}_{d}$ et $\Omega_{E, N}=\operatorname{Im}\left(\omega_{1} \bar{\omega}_{2}\right)$ est le volume complexe de $E$ (c'est-à-dire le double de l'aire d'un parallélogramme fondamental de $E(\mathbb{C})$ ). 
Nous rappelons finalement la relation évidente

$$
\hat{h}_{\mathbb{K}_{d}}\left(\operatorname{Tr}_{d}\right)=\hat{h}_{\mathbb{H}_{d}}\left(\mathrm{P}_{d}\right)+\sum_{\sigma \in G_{d} \backslash\{I d\}}<\mathrm{P}_{d}, \mathrm{P}_{d}^{\sigma}>_{\mathbb{H}_{d}}
$$

entre nos deux objets d'étude $(<\cdot, \cdot\rangle_{\mathbb{H}_{d}}$ désigne la forme bilinéaire de Néron-Tate sur $\mathbb{H}_{d}$ ). Le troisième terme représente l'angle formé par les points de Heegner entre eux et il sera intéressant de l'analyser (voir paragraphe 5 ).

\section{LES TRACES}

3.1. Mise en place. Il est ici nécessaire de raisonner selon le rang de la courbe $E$. En effet, soit $L(E \mid \mathbb{Q}, s):=\sum_{n \geqslant 1} a_{n} n^{-s}$ sa série $L$ sur $\mathbb{Q}$ définie a priori sur $\Re(s)>\frac{3}{2}$. Selon les travaux de A. Wiles et de R. Taylor ([Wi] et TaWi]), il existe une forme primitive cuspidale $f$ de niveau $N$, de poids 2 et de caractère trivial telle que

$$
L(E \mid \mathbb{Q}, s)=L(f, s)
$$

sur $\Re(s)>\frac{3}{2}$. Par conséquent, $L(E \mid \mathbb{Q}, s)$ admet un prolongement holomorphe à $\mathbb{C}$ et satisfait l'équation fonctionnelle

$$
\left(\frac{\sqrt{N}}{2 \pi}\right)^{s} \Gamma(s) L(E \mid \mathbb{Q}, s)=\omega\left(\frac{\sqrt{N}}{2 \pi}\right)^{2-s} \Gamma(2-s) L(E \mid \mathbb{Q}, 2-s)
$$

où $\omega= \pm 1$ est une valeur propre d'Atkin-Lehner de $f$. Définissons pour tout discriminant $d$ dans $\mathcal{D}$ la fontion $L$ de $E$ sur $\mathbb{Q}$ tordue par $\chi_{d} \operatorname{sur} \Re(s)>\frac{3}{2}$ par

$$
L\left(E \mid \mathbb{Q} \times \chi_{d}, s\right):=\sum_{n \geqslant 1} \frac{a_{n} \chi_{d}(n)}{n^{s}} .
$$

$L\left(E \mid \mathbb{Q} \times \chi_{d}, s\right)$ admet un prolongement holomorphe à $\mathbb{C}$ et satisfait l'équation fonctionnelle

$$
\left(\frac{|d| \sqrt{N}}{2 \pi}\right)^{s} \Gamma(s) L\left(E \mid \mathbb{Q} \times \chi_{d}, s\right)=\omega_{d}\left(\frac{|d| \sqrt{N}}{2 \pi}\right)^{2-s} \Gamma(2-s) L\left(E \mid \mathbb{Q} \times \chi_{d}, 2-s\right)
$$

où $\omega_{d}:=\omega \chi_{d}(-N)=-\omega($ voir $[\mathrm{wK}])$. Avec ces notations, la factorisation

$$
L\left(E \mid \mathbb{K}_{d}, s\right)=L(E \mid \mathbb{Q}, s) L\left(E \mid \mathbb{Q} \times \chi_{d}, s\right)
$$

est valide (voir $[\mathrm{Da}]$ ) d'où

$$
L^{\prime}\left(E \mid \mathbb{K}_{d}, 1\right)=L^{\prime}(E \mid \mathbb{Q}, 1) L\left(E \mid \mathbb{Q} \times \chi_{d}, 1\right)+L(E \mid \mathbb{Q}, 1) L^{\prime}\left(E \mid \mathbb{Q} \times \chi_{d}, 1\right) .
$$

Ainsi, l'étude de la hauteur des traces des points de Heegner en moyenne sur les discriminants $d$ dans $\mathcal{D}$ est ramenée à l'étude de la valeur au point critique des fonctions $L$ tordues lorsque $E$ est de rang analytique 1 et à l'étude des dérivées au point critique des fonctions $L$ tordues lorsque $E$ est de rang analytique 0 car alors $\omega=+1$. 
3.2. Courbes de rang analytique 0. Rappelons le théorème obtenu par H. Iwaniec dans $[\mathrm{Iw}]$. Avant cela, fixons une fois pour toutes les deux notations

$$
\gamma(4 N):=\#\left\{d \bmod 4 N, d \equiv \nu^{2} \bmod 4 N,(\nu, 4 N)=1\right\},
$$

et

$$
c_{N}:=\frac{3 \gamma(4 N)}{\pi^{2} N} \prod_{\substack{p \in \mathcal{P} \\ p \mid 2 N}}\left(1-\frac{1}{p^{2}}\right)^{-1}
$$

Théorème 3.1. Si $E$ une courbe elliptique rationnelle de conducteur $N$ sans facteurs carrés et de rang analytique 0 et $F$ est une fonction lisse à support compact dans $\mathbb{R}_{+}$ et de moyenne strictement positive alors

$$
\sum_{d \in \mathcal{D}} L^{\prime}\left(E \mid \mathbb{Q} \times \chi_{d}, 1\right) F\left(\frac{|d|}{Y}\right)=\alpha_{N} Y \log Y+\beta_{N} Y+\mathcal{O}_{\varepsilon}\left(N^{\frac{23}{14}+\varepsilon} Y^{\frac{13}{14}+\varepsilon}\right)
$$

pour tout $\varepsilon>0$ où

$$
\alpha_{N}:=c_{N} L(1) \int_{0}^{+\infty} F(t) \mathrm{d} t \neq 0
$$

et

$$
\beta_{N}:=c_{N} \int_{0}^{+\infty} F(t)\left(L^{\prime}(1)+L(1)\left(\log \left(\frac{\sqrt{N} t}{2 \pi}\right)-\gamma\right)\right) \mathrm{d} t
$$

avec

$$
L(s):=\frac{L\left(S y m^{2} E, 2 s\right)}{\zeta^{(N)}(4 s-2)}\left(\prod_{\substack{p \in \mathcal{P} \\ p \mid N}}\left(1-\frac{a_{p}}{p^{s}}\right)^{-1}\left(1-\frac{a_{p^{2}}}{p^{2 s}}\right)\right) \mathcal{P}(s)
$$

et

$$
\mathcal{P}(s):=\prod_{\substack{p \in \mathcal{P} \\ p \nmid 2 N}}\left(\frac{1}{1+1 / p}+\left(\frac{1}{1+p}\right)\left(\frac{1+p^{2-4 s}-\left(a_{p}^{2}-2 p\right) p^{-2 s}}{1+p^{1-2 s}}\right)\right) .
$$

Remarque 3.1. Dans $\underline{\mathrm{Iw}}$, la fonction $L$ est définie par la série de Dirichlet suivante

$$
L(s)=\sum_{\substack{n=k \ell^{2} \\ k \mid N^{\infty} \\(\ell, N)=1}} \frac{b_{n}}{n^{s}}
$$

avec

$$
b_{n}:=a_{n} \prod_{\substack{p \in \mathcal{P} \\ p \mid n \\ p \nmid 2 N}}\left(1+\frac{1}{p}\right)^{-1}
$$


pour tout entier naturel non-nul $n$. Celle-ci peut se réécrire sous la forme

$$
L(s)=\prod_{\substack{p \in \mathcal{P} \\ p \mid N}}\left(1-\frac{a_{p}}{p^{s}}\right)^{-1} \prod_{\substack{p \in \mathcal{P} \\ p \nmid 2 N}}\left(1+\left(1+\frac{1}{p}\right)^{-1}\left(\sum_{i=1}^{\infty} \frac{a_{p^{2 i}}}{p^{2 i s}}\right)\right) \prod_{\substack{p \in \mathcal{P} \\ p \mid(2, N-1)}}\left(1+\sum_{i=1}^{\infty} \frac{a_{p^{2 i}}}{p^{2 i s}}\right)
$$

ce qui permet de retrouver l'expression de $L$ donnée dans le théorème en fonction du carré symétrique de $E$ et du produit Eulérien $\mathcal{P}$. Signalons que l'holomorphie de la fonction $L\left(\mathrm{Sym}^{2} E, s\right)$ dans tout le plan complexe a été prouvée par Shimura dans [Sh] et que le produit Eulérien $\mathcal{P}(s)$ est absolument convergeant sur $\Re(s)>\frac{3}{4}$ et y définit une fonction holomorphe.

Remarque 3.2. La valeur de la fonction $L\left(\operatorname{Sym}^{2} E, s\right)$ au bord de la bande critique est reliée au degré de la paramétrisation modulaire $\Phi_{N, E}: X_{0}(N) \rightarrow E$ de $E$ (cf. Wa (1-1)) par la formule suivante analogue à celle du nombre de classes de Dirichlet

$$
\frac{L\left(\operatorname{Sym}^{2} E, 2\right)}{\pi \Omega_{E, N}}=\frac{\operatorname{deg}\left(\Phi_{N, E}\right)}{N c_{E}(N)^{2}}
$$

où $c_{E}(N)$ est la $\Gamma_{0}(N)$-constante de Manin de $E$ qui est un entier relatif uniformément borné $(\underline{\mathrm{Ed}}])$. Ici, on utilise le fait que $N$ est sans facteurs carrés à deux reprises :

- les fonctions $L$ du carré symétrique de $E$ motivique et analytique coincident car il n'y a pas de termes correctifs en les nombres premiers dont le carré divise le conducteur $N$,

- lorsque $E$ est une courbe de Weil $X_{0}(N)$-forte et $N$ est impair, la constante de Manin vaut \pm 1 selon les travaux de A. Abbes et E. Ullmo ( Manin ( $\underline{\mathrm{Ma}})$ a conjecturé que cette constante vaut \pm 1 pour toute courbe de Weil $X_{0}(N)$-forte $^{2}$.

Remarque 3.3. Il semblerait que quelques petites erreurs de frappe dans $\alpha_{N}$ (et en fait dans $c_{N}$ et $L$ ) se soient glissées dans $[\mathrm{Iw}$. La valeur donnée ici est corrigée.

Remarque 3.4. Dans Iw, la dépendance en le conducteur $N$ de la courbe dans le terme d'erreur n'est pas explicite. Cependant, il suffit de reprendre les différentes majorations pour restituer celle-ci.

Corollaire 3.2. Si E est une courbe elliptique rationnelle de conducteur $N$ sans facteurs carrés et de rang analytique 0 alors

$$
\begin{aligned}
& \text { (3.6) } \sum_{\substack{d \in \mathcal{D} \\
|d| \leqslant Y}} \hat{h}_{\mathbb{K}_{d}}\left(\operatorname{Tr}_{d}\right)=C_{\operatorname{Tr}}^{(0)} Y^{\frac{3}{2}} \log Y+\frac{C_{\operatorname{Tr}}^{(0)}}{2} \log (N) Y^{\frac{3}{2}} \\
& +\frac{L(E \mid \mathbb{Q}, 1) c_{N}}{3 \Omega_{E, N}}\left(L^{\prime}(1)-L(1)\left(\frac{2}{3}+\log (2 \pi)+\gamma\right)\right) Y^{\frac{3}{2}}+\mathcal{O}_{\varepsilon}\left(\frac{L(E \mid \mathbb{Q}, 1)}{\Omega_{E, N}} N^{\frac{23}{14}+\varepsilon} Y^{\frac{20}{14}}+\varepsilon\right)
\end{aligned}
$$

\footnotetext{
${ }^{2}$ Ceci est faux si $E$ n'est pas forte : [0,1,1,0,0] a une $X_{0}(11)$-constante de Manin égale à 5 .
} 
pour tout $\varepsilon>0$ où $C_{\mathrm{Tr}}^{(0)}$ est la constante définie par

$$
C_{\mathrm{Tr}}^{(0)}:=\frac{2}{\pi} c_{N} \mathcal{P}(1) L(E \mid \mathbb{Q}, 1) \frac{L\left(S y m^{2} E, 2\right)}{\pi \Omega_{E, N}} L_{E}
$$

en notant $L_{E}$ un produit de facteurs locaux correspondant aux facteurs Eulériens de $L(E \mid \mathbb{Q}, s)$ et de $L\left(\operatorname{Sym}^{2}(E), s\right)$ en les nombres premiers divisant le conducteur

$$
L_{E}:=\prod_{\substack{p \in \mathcal{P} \\ p \mid N}}\left(1-\frac{a_{p}}{p}\right)^{-1}\left(1-\frac{1}{p^{2}}\right)^{-1}\left(1-\frac{a_{p^{2}}}{p^{2}}\right) .
$$

Preuve du corollaire 3.2. Il suffit d'appliquer la formule de Gross-Zagier (2.3) et de prendre pour $F$ une approximation lisse de la fonction qui vaut $\frac{3}{2} \sqrt{t}$ sur $[0,1]$ et 0 en dehors de cet intervalle. Le corollaire découle alors de (3.1).

Remarque 3.5. À conducteur $N$ fixé, le terme principal dans (3.6) est $C_{T r}^{(0)} Y^{\frac{3}{2}} \log Y$ avec

$$
C_{T r}^{(0)}=\left(\frac{6}{\pi^{3} c_{E}(N)^{2}} \mathcal{P}(1) \prod_{\substack{p \in \mathcal{P} \\ p \mid 2 N}}\left(1-\frac{1}{p^{2}}\right)^{-1}\right) \times L(E \mid \mathbb{Q}, 1) L_{E} \times \frac{\gamma(4 N)}{N^{2}} \operatorname{deg}\left(\Phi_{N, E}\right)
$$

et est donc proportionnel au degré de la paramétrisation modulaire. Par contre, si $N=$ $Y^{a}$ avec $0<a<\frac{1}{23}$ alors le deuxième terme de (3.6) est du même ordre de grandeur que le premier et le terme principal devient

$$
\left(1+\frac{a}{2}\right) C_{T r}^{(0)} Y^{\frac{3}{2}} \log Y
$$

C'est la principale raison pour laquelle nous avons rendu explicite la dépendance en le conducteur $N$ de la courbe dans le terme d'erreur. Il serait aussi intéressant d'étudier l'influence des valeurs extrémales de $L(E \mid \mathbb{Q}, 1)$ sur la moyenne des hauteurs des traces des points de Heegner. Malheureusement, cela ne semble pas vérifiable numériquement étant donné le temps de calcul nécessaire par les algorithmes à utiliser.

Le produit Eulérien $\mathcal{P}(s)$ varie peu. En effet, si l'on effectue un développement limité du facteur local en le nombre premier $p$ de $\mathcal{P}(1)$, on obtient

$$
\mathcal{P}_{p}(1)=1-\frac{a_{p}^{2}-2 p}{p^{3}}+\mathcal{O}\left(\frac{1}{p^{2}}\right)
$$

d'où l'existence d'une constante absolue $C>0$ ne dépendant pas de la courbe $E$ considérée telle que

$$
C \prod_{p \in \mathcal{P}}\left(1-\frac{2}{p^{2}}\right)<\mathcal{P}(1)<C \prod_{p \in \mathcal{P}}\left(1+\frac{2}{p^{2}} .\right)
$$


Finalement, les termes importants sont le degré de la paramétrisation modulaire, la valeur de la fonction $L$ de la courbe au point critique 1 ainsi que le conducteur qui intervient en $\gamma(4 N) / N^{2}$. À conducteur et degré fixés, les petits nombres premiers qui

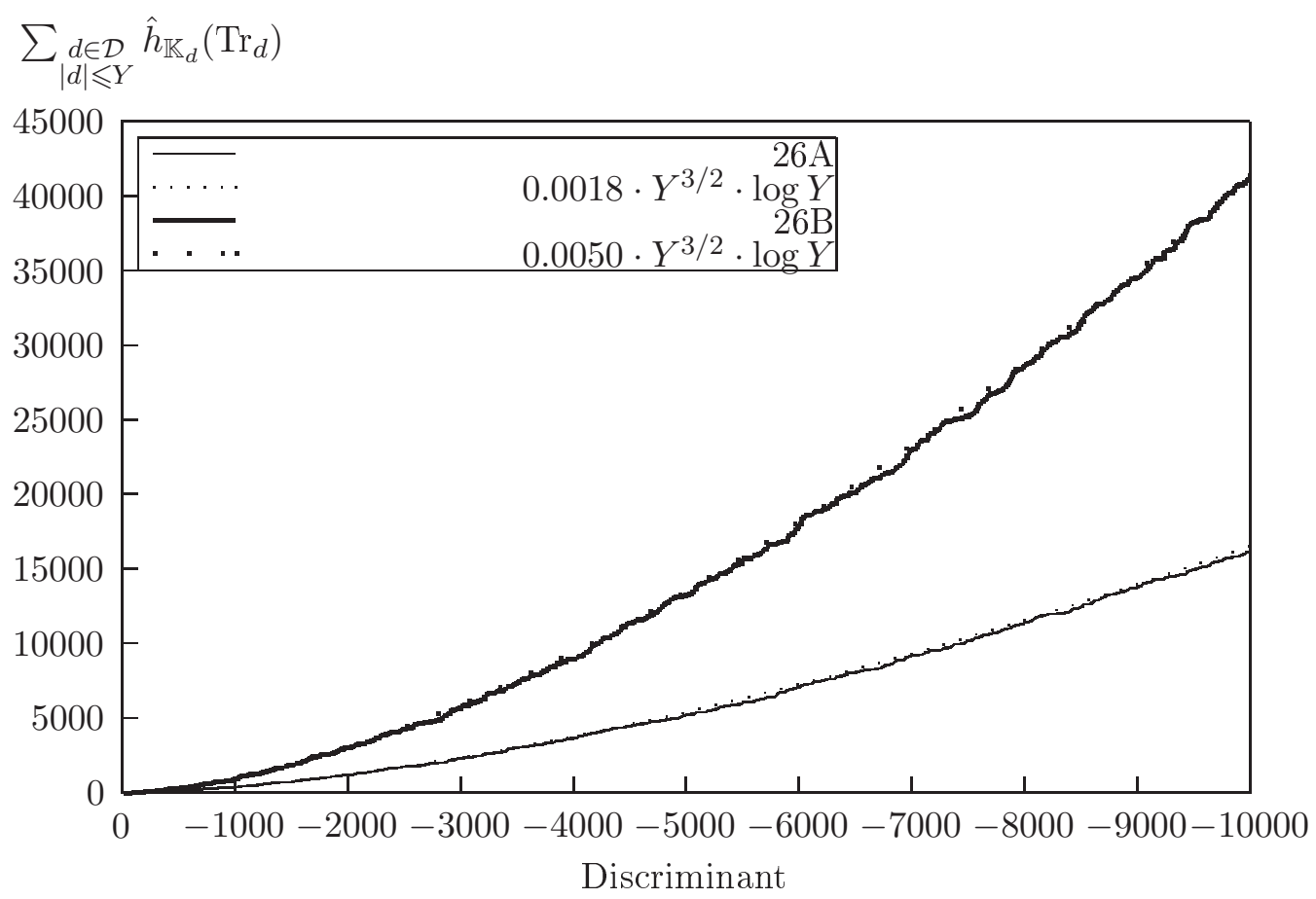

Fig. 2 - Hauteur des traces en moyenne sur les courbes $26 \mathrm{~A}$ et $26 \mathrm{~B}$ : pratiques et théoriques.

divisent le conducteur ont un rôle décisif et les hauteurs des traces ont alors tendance à être plus ou moins grandes suivant que $E$ a réduction multiplicative déployée ou nondéployée en ces nombres premiers. Cette influence se voit très bien dans le cas des deux courbes de conducteur 26 (de rang analytique 0) dont les paramétrisations modulaires ont même degré 2 alors que les traces sont presque trois fois plus grosses sur la 26B que sur la 26A. Ceci s'explique analytiquement par le fait que 26 est divisible par 2 et que la $26 \mathrm{~A}$ a réduction multiplicative déployée en 2 alors que la 26B a réduction multiplicative non-déployée. Ceci induit un facteur 3 entre les produits pour les nombres premiers $p$ divisant le conducteur de ces courbes des facteurs Euleriens de leur fonction $L$ en $p$. La figure 2 illustre cette différence de comportement. On voit également que la courbe représentant la somme des hauteurs des traces est très proche de la courbe théorique même si elle est relativement irrégulière. Rappelons que 0.0018 et 0.0050 sont les valeurs 
numériques de la constante $C_{\mathrm{Tr}}^{(0)}$ apparaissant dans le corollaire 3.2 pour les courbes $26 \mathrm{~A}$ et $26 \mathrm{~B}$.

3.3. Courbes de rang analytique 1. Lorsque l'on considère une courbe de rang 1 , on est amené à estimer la moyenne des valeurs en 1 des fonctions $L$ tordues et non de leurs dérivées.

Théorème 3.3. Si $E$ est une courbe elliptique rationnelle de conducteur $N$ sans facteurs carrés et de rang analytique 1 et $F$ est une fonction lisse à support compact dans $\mathbb{R}_{+}$ et de moyenne strictement positive alors

$$
\sum_{d \in \mathcal{D}} L\left(E \mid \mathbb{Q} \times \chi_{d}, 1\right) F\left(\frac{|d|}{Y}\right)=\alpha_{N} Y+\mathcal{O}_{\varepsilon}\left(N^{\frac{23}{14}+\varepsilon} Y^{\frac{13}{14}+\varepsilon}\right)
$$

pour tout $\varepsilon>0$ où $\alpha_{N}$ est définie en (3.4).

Idée de preuve du théorème [3.3. Il n'est pas difficile d'adapter la démonstration de $[\mathrm{Iw}$ à ce cas. En reprenant les notations de l'article, il suffit de remplacer la fonction $V(X)$ définie au paragraphe 4 page 369 par la fonction

$$
\tilde{V}(X)=e^{-X} \text {. }
$$

On a alors $^{3}$,

$$
\mathcal{A}\left(X, \chi_{d}\right)=\frac{1}{2 i \pi} \int_{(3 / 4)} L\left(s+1, E, \chi_{d}\right) \Gamma(s)\left(\frac{X}{2 \pi}\right)^{s} d s .
$$

La majoration $\mathcal{A}\left(X, \chi_{d}\right) \ll \sqrt{X}$, qui découle de l'inégalité de Hölder et d'une estimation des $a_{n}$ tient toujours, et ainsi les majorations successives effectuées dans la démonstration ne posent pas de problème. La seule différence notable vient à la page 374 lors du calcul de $\mathcal{B}(X)$. On a alors

$$
\operatorname{res}_{s=0} L(s+1) \Gamma(s)\left(\frac{X}{2 \pi}\right)^{s}=L(1)
$$

et il n'apparaît pas de terme en $\log X$.

On en déduit comme pour le rang 0 le corollaire suivant.

Corollaire 3.4. Si E est une courbe elliptique rationnelle de conducteur $N$ sans facteurs carrés et de rang analytique 1 alors

$$
\sum_{\substack{d \in \mathcal{D} \\|d| \leqslant Y}} \hat{h}_{\mathbb{K}_{d}}\left(\operatorname{Tr}_{d}\right)=C_{\operatorname{Tr}}^{(1)} Y^{3 / 2}+\mathcal{O}_{\varepsilon}\left(\frac{L(E \mid \mathbb{Q}, 1)}{\Omega_{E, N}} N^{\frac{23}{14}+\varepsilon} Y^{\frac{20}{14}+\varepsilon}\right)
$$

\footnotetext{
${ }^{3} \grave{A}$ noter une erreur de frappe dans $\underline{\underline{I w}}$, il s'agit bien de $\left(\frac{X}{2 \pi}\right)^{s}$ et non de son inverse.
} 
pour tout $\varepsilon>0$ où $C_{\mathrm{Tr}}^{(1)}$ est la constante définie par

$$
C_{\operatorname{Tr}}^{(1)}:=\frac{2}{\pi} c_{N} \mathcal{P}(1) L^{\prime}(E \mid \mathbb{Q}, 1) \frac{L\left(S y m^{2} E, 2\right)}{\pi \Omega_{E, N}} L_{E}
$$

où $c_{N}$ est définie en (3.3), $L_{E}$ en (3.7) et $\mathcal{P}$ en (3.5).

Remarque 3.6. À conducteur $N$ fixé, le terme principal dans (3.4) est $C_{T r}^{(1)} Y^{\frac{3}{2}}$ avec

$$
C_{T r}^{(1)}=\left(\frac{6}{\pi^{3} c_{E}(N)^{2}} \mathcal{P}(1) \prod_{\substack{p \in \mathcal{P} \\ p \mid 2 N}}\left(1-\frac{1}{p^{2}}\right)^{-1}\right) \times L^{\prime}(E \mid \mathbb{Q}, 1) L_{E} \times \frac{\gamma(4 N)}{N^{2}} \operatorname{deg}\left(\Phi_{N, E}\right)
$$

et est donc proportionnel au degré de la paramétrisation modulaire.

Remarque 3.7. Il est intuitivement étonnant que les traces en moyennes des points de Heegner sur une courbe elliptique $E$ sont asymptotiquement plus grosses par un facteur logarithmique si le rang de la courbe elliptique est minimal.

Les deux courbes elliptiques de conducteur 91 ont même rang 1 et on a représenté sur la figure 3 les hauteurs des traces $\operatorname{Tr}_{d}$ sur ces deux courbes ainsi que la courbe théorique donnée par le corollaire ci-dessus. On constate que les courbes sont beaucoup plus irrégulières que dans le cas du rang 0 (figure 20) même si elles suivent la courbe théorique de très près. La figure 4 illustre les différences de croissance des hauteurs des traces sur les courbes 37A (rang 1) et 37B (rang 0). On remarque que le comportement est très irrégulier. Ceci est en partie dû à la division par $Y^{3 / 2}$ qui rend les irrégularités plus apparentes que dans la figure 2. De plus, pour la courbe 37A de rang 1, il arrive fréquemment que la trace soit nulle ce qui «casse la moyenne». Il est conjecturé que la proportion d'annulation de $L\left(E \mid \mathbb{Q} \times \chi_{d}, 1\right)$ (ce qui correspond aux cas de trace nulle selon la formule de Gross-Zagier et la conjecture de Birch et Swinnerton-Dyer) tend vers 0 lorsque le discriminant tend vers l'infini ${ }^{4}$ mais cela ne se voit pas dans l'échelle de discriminants étudiée.

\section{Estimation asymptotique de la hauteur des points De HeEgner}

Nous démontrons une formule asymptotique pour les hauteurs des points de Heegner $\mathrm{P}_{d}$ semblable à celle que nous avons donnée pour les traces. Soient $E$ une courbe elliptique définie sur $\mathbb{Q}$, de conducteur $N$ et $L(E \mid \mathbb{Q}, s):=\sum_{n \geqslant 1} a_{n} n^{-s}$ sa fonction $L$ (de rang analytique quelconque). On s'intéresse à la valeur moyenne des dérivées en 1 des séries de Dirichlet $L_{d}(E, s)$ définies par (2.1).

\footnotetext{
${ }^{4}$ Le type de symétrie de cette famille de fonctions $L$ est orthogonal impair.
} 
Théorème 4.1. Si E est une courbe elliptique rationnelle de conducteur $N$ sans facteurs carrés et de rang analytique quelconque et $F$ est une fonction lisse à support compact dans $\mathbb{R}_{+}$et de moyenne strictement positive alors

$$
\sum_{d \in \mathcal{D}} L_{d}^{\prime}(E, 1) F\left(\frac{|d|}{Y}\right)=\widetilde{\alpha_{N}} Y \log Y+\widetilde{\beta_{N}} Y+\text { Error }+\mathcal{O}_{\varepsilon}\left(N^{\frac{15}{4}+\varepsilon} Y^{\frac{19}{20}+\varepsilon}\right)
$$

où

$$
\text { Error }=\mathcal{O}_{\varepsilon}\left(N Y(\log (N Y))^{\frac{1}{2}+\varepsilon}\right)
$$

pour tout $\varepsilon>0$ et où

$$
\widetilde{\alpha_{N}}:=c_{N} \widetilde{L}(1) \int_{0}^{+\infty} F(t) \mathrm{d} t \neq 0
$$

et

$$
\widetilde{\beta_{N}}:=c_{N} \int_{0}^{+\infty} F(t)\left(\widetilde{L}^{\prime}(1)+\widetilde{L}(1)\left(\log \left(\frac{N t}{4 \pi^{2}}\right)-2 \gamma\right)\right) \mathrm{d} t
$$

$\sum_{\substack{d \in \mathcal{D} \\|d| \leqslant Y}} \hat{h}_{\mathbb{K}_{d}}\left(\operatorname{Tr}_{d}\right)$

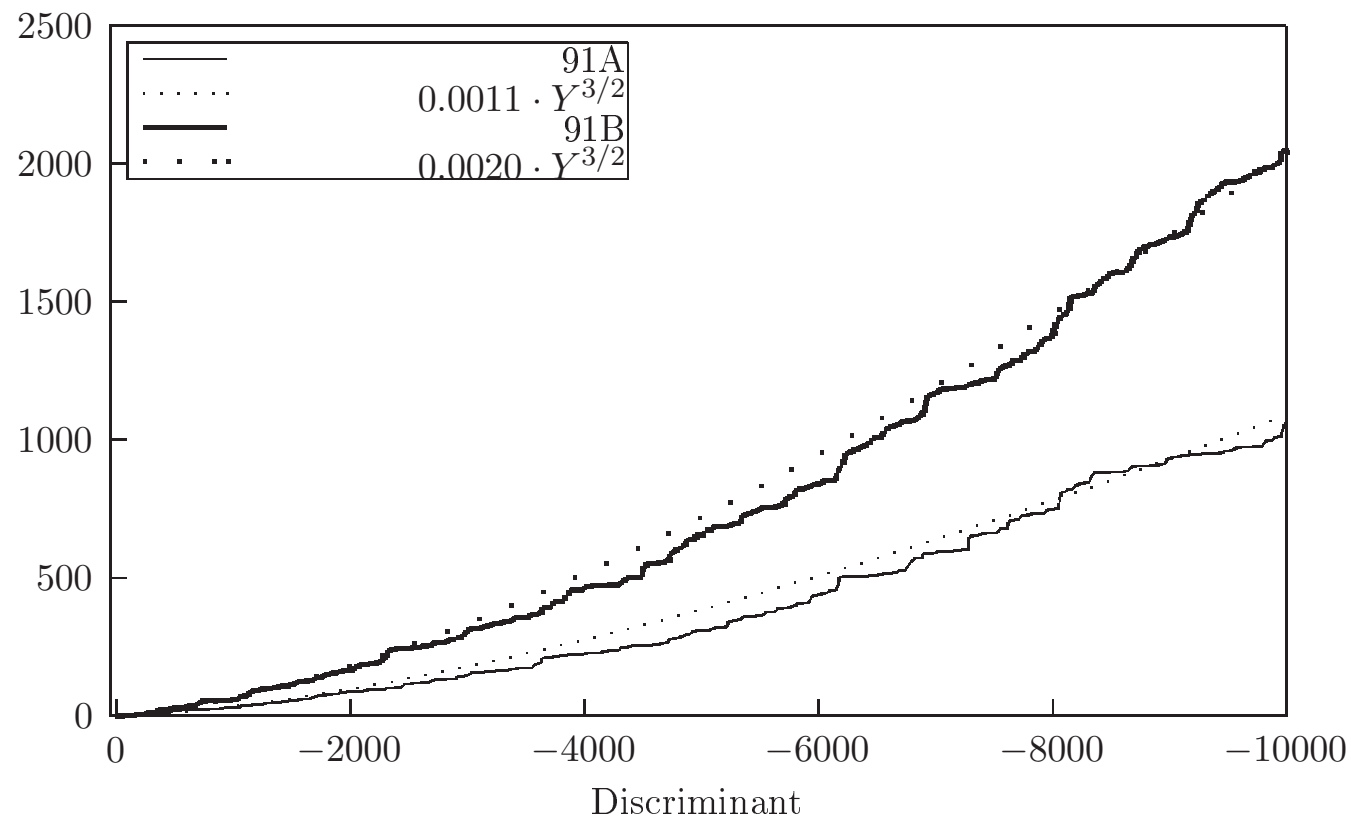

FIG. 3 - Hauteur des traces en moyenne sur les courbes 91A et 91B. 


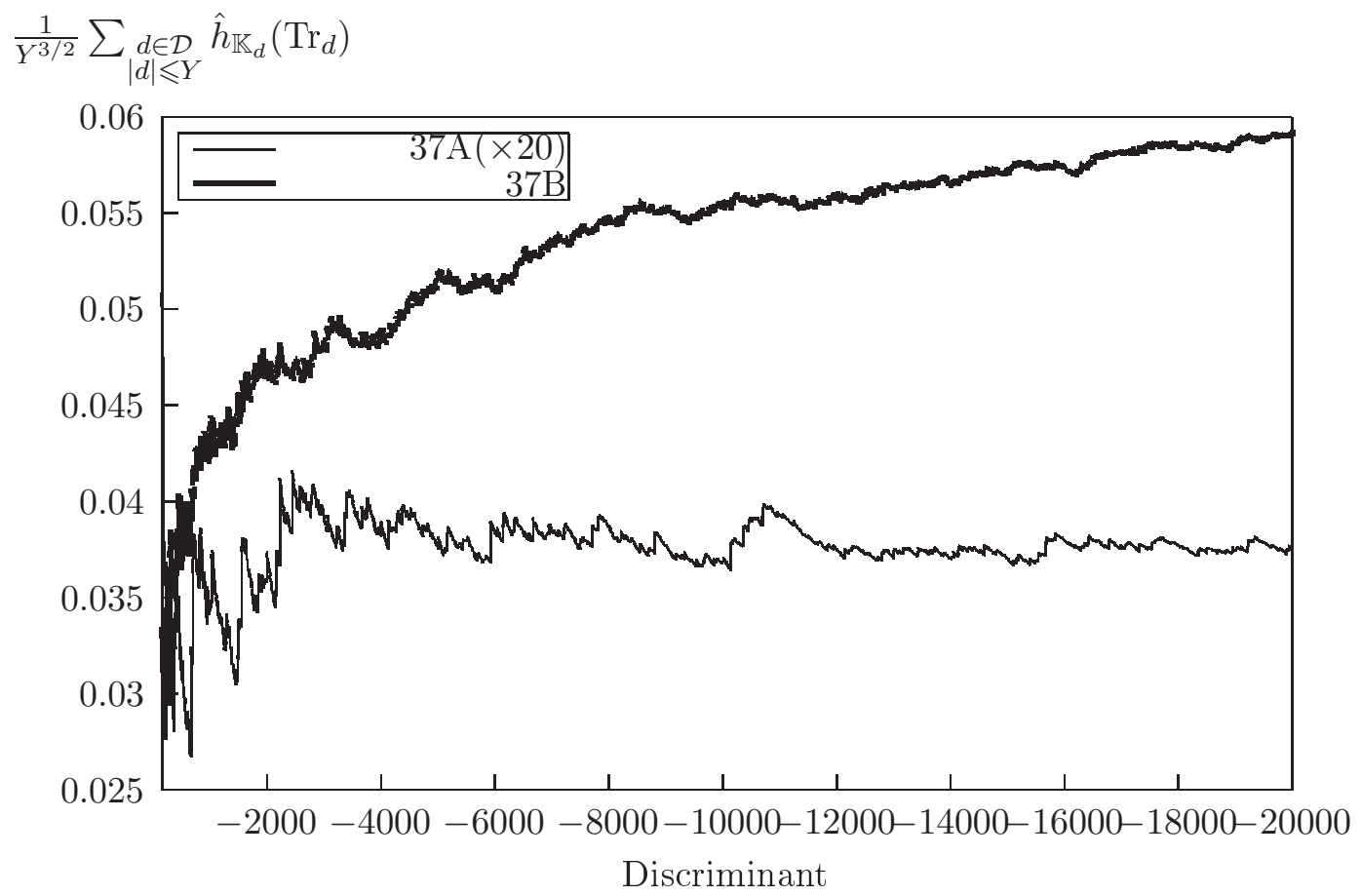

FIG. 4 - Hauteur des traces en moyenne sur les courbes 37A et 37B.

où la constante $c_{N}$ est définie en (3.3) avec

$$
\widetilde{L}(s):=\frac{L\left(S_{\left.y m^{2} E, 2 s\right)}\right.}{\zeta^{(N)}(2 s)} \widetilde{\mathcal{P}}(s) \times \begin{cases}\frac{4}{3} & \text { si } N \text { est impair } \\ 1 & \text { sinon }\end{cases}
$$

et

$$
\widetilde{\mathcal{P}}(s):=\prod_{\substack{p \in \mathcal{P} \\ p \nmid 2 N}}\left(1+\left(1+\frac{1}{p}\right)^{-1}\left(p^{4 s-2}-1\right)^{-1}\right) .
$$

Remarque 4.1. À conducteur $N$ fixé, il semble que l'on obtienne un développement asymptotique du premier moment par rapport à $Y$ à un seul terme et non à deux termes comme dans le Théorème 3.1 de H. Iwaniec. Cependant, les résultats numériques décrits dans la dernière partie du paragraphe 5 et notre intuition analytique nous permettent de conjecturer que

$$
\text { Error }=o_{\varepsilon}(N Y)
$$


Pour pouvoir prouver cela, il faudrait notamment être en mesure de déterminer le comportement asymptotique de moyennes de la forme

$$
\sum_{\substack{1 \leqslant u \leqslant U \\ 1 \leqslant v \leqslant V}} \sum_{d \in \mathcal{D}} a_{u, v} \chi_{d}(u) r_{d}(v) F\left(\frac{|d|}{Y}\right)
$$

pour tous nombres réels strictement positifs $U, V$ et toute suite de nombres complexes $\left(a_{u, v}\right)_{\substack{1 \leqslant u \leqslant U \\ 1 \leqslant v \leqslant V}}$ (se reporter également en page 26). Les auteurs projettent de s'intéresser dans un avenir proche à ce type de moyennes qui sont en réalité un cas particulier de quantités beaucoup plus générales. En outre, il ne fait aucun doute que la dépendance en $N$ dans $\mathcal{O}_{\varepsilon}\left(N^{\frac{15}{4}+\varepsilon} Y^{\frac{19}{20}+\varepsilon}\right)$ peut être améliorée en étant plus soigneux mais une croissance au plus polynomiale en le conducteur nous suffit.

Preuve du théorème 4.1 B.H. Gross et D. Zagier (GrZa ) ont prouvé que la série de Dirichlet $L_{d}(E, s)$ définie a priori sur $\Re(s)>\frac{3}{2}$ admet un prolongement holomorphe à $\mathbb{C}$ et satisfaisait l'équation fonctionnelle

$$
\forall s \in \mathbb{C}, \quad \Lambda_{d}(E, s)=-\chi_{d}(N) \Lambda_{d}(E, 2-s)
$$

où $\Lambda_{d}(E, s):=(N|d|)^{s}\left((2 \pi)^{-s} \Gamma(s)\right)^{2} L_{d}(E, s)$ est la série de Dirichlet complétée. Remarquons que comme $d$ est un carré modulo $N$, le signe de l'équation fonctionnelle vaut -1 d'où $L_{d}(E, 1)=0$. Ceci va nous permettre d'exprimer $L_{d}^{\prime}(E, 1)$ en terme de deux sommes convergeant exponentiellement vite en suivant une procédure analytique désormais classique (confer Théorème 5.3. de [wKo] pour plus de détails). Pour $X>0$, posons

$$
V(X):=\frac{1}{2 i \pi} \int_{(3 / 4)} \Gamma(s)^{2} X^{-s} d s
$$

et

$$
\mathcal{A}_{d}(E, X):=\frac{1}{2 i \pi} \int_{(3 / 4)} L_{d}(E, s+1) \Gamma(s)^{2}\left(\frac{X}{4 \pi^{2}}\right)^{s} d s .
$$

Le développement de $L_{d}(E, s)$ en série de Dirichlet absolument convergente $\operatorname{sur} \Re(s)>\frac{3}{2}$ assure que

$$
\mathcal{A}_{d}(E, X)=\sum_{n=1}^{\infty} \frac{a_{n} r_{d}(n)}{n} \sum_{(m, N)=1} \chi_{d}(m) \frac{1}{m} V\left(\frac{4 \pi^{2} n m^{2}}{X}\right) .
$$

On déplace la ligne d'intégration jusqu'à $\Re(s)=-3 / 4$ croisant un unique pole en $s=0$ de résidu égal à $L_{d}^{\prime}(E, 1)$ puis on revient en $s=3 / 4$ par le changement de variables $s \mapsto-s$. L'équation fonctionnelle entraîne alors que

$$
L_{d}^{\prime}(E, 1)=\mathcal{A}_{d}(E, X)+\mathcal{A}_{d}\left(E, \frac{(N d)^{2}}{X}\right)
$$

et en particulier que

$$
L_{d}^{\prime}(E, 1)=2 \mathcal{A}_{d}(E,|d| N) .
$$


Bornons $V(X)$ pour $X>0$ de la manière suivante :

$$
\begin{aligned}
V(X) & =\frac{1}{2 i \pi} \int_{u=0}^{\infty} \frac{e^{-u}}{u} \int_{v=0}^{\infty} e^{-v}\left(\int_{(3 / 4)}\left(\frac{u v}{X}\right)^{s} \frac{1}{s} d s\right) d v d u \\
& \ll \int_{0}^{\infty} \frac{e^{-(u+X / u)}}{u} d u \\
& \ll X^{-1 / 4} \exp (-2 \sqrt{X})
\end{aligned}
$$

et en fait $X^{j} V^{(j)}(X) \ll_{j} X^{-1 / 4} \exp (-2 \sqrt{X})$ pour tout entier naturel $j$. Posons

$$
S_{N}(Y):=\sum_{d \in \mathcal{D}} L_{d}^{\prime}(E, 1) F\left(\frac{|d|}{Y}\right) .
$$

Comme $d$ est dans $\mathcal{D}, d$ est un carré modulo 4 et est premier à 4 donc $d$ est congru à 1 modulo 4. Ainsi, $\mathcal{O}_{d}=\mathbb{Z}+\frac{1+\sqrt{d}}{2} \mathbb{Z}$ et un calcul élémentaire montre que

$$
r_{d}(n)=\#\left\{(u, v) \in\left(\mathbb{N}^{*} \times \mathbb{Z}\right) \cup(\{0\} \times \mathbb{N}), u^{2}+|d| v^{2}=4 n\right\} .
$$

On observe que si $n$ est un carré alors $(2 \sqrt{n}, 0)$ est une solution de l'équation ci-dessus alors qu'il n'existe pas de solutions de la forme $(0, *)$ ce qui prouve que

$r_{d}(n)=1+\#\left\{v \in \mathbb{Z}^{*},|d| v^{2}=4 n\right\}+\#\left\{(u, v) \in\left(\mathbb{N}^{*} \times \mathbb{Z}^{*}\right), u^{2}+|d| v^{2}=4 n\right\}:=1+r_{d}^{\prime}(n)$

et que

$$
\sum_{\substack{d \in \mathcal{D} \\ Y \ll|d| \ll Y}} r_{d}(n) \geqslant \#\{d \in \mathcal{D}, Y \ll|d| \ll Y\} \sim_{Y \rightarrow+\infty} C Y
$$

pour une constante absolue $C>0$. Si $n$ n'est pas un carré alors $r_{d}(n)=r_{d}^{\prime}(n)$. Dans chacun des cas, si $(u, v)$ est une solution contribuant à $r_{d}^{\prime}(n)$ pour un $d$ inférieur à $Y$ dans $\mathcal{D}$ alors $u \leqslant 2 \sqrt{n}$ et à chaque tel $u$ correspond au plus deux couples $(d, v)$ (car $d$ est supposé sans facteurs carrés) d'où

$$
\sum_{\substack{d \in \mathcal{D} \\ Y \ll|d| \ll Y}} r_{d}^{\prime}(n) \leqslant 4 \sqrt{n}
$$

On écrit alors ${ }^{5} S_{N}(Y):=\mathrm{TP}_{1}+\operatorname{Err}_{1}$ où

$$
\mathrm{TP}_{1}:=2 \sum_{n \geqslant 1} \frac{a_{n^{2}}}{n^{2}} \sum_{(m, N)=1} \frac{1}{m} \sum_{d \in \mathcal{D}} \chi_{d}(m) V\left(\frac{4 \pi^{2} n^{2} m^{2}}{N|d|}\right) F\left(\frac{|d|}{Y}\right)
$$

et

$$
\operatorname{Err}_{1}:=2 \sum_{d \in \mathcal{D}} \sum_{n \geqslant 1} \frac{a_{n} r_{d}^{\prime}(n)}{n} \sum_{(m, N)=1} \frac{1}{m} \chi_{d}(m) V\left(\frac{4 \pi^{2} n m^{2}}{N|d|}\right) F\left(\frac{|d|}{Y}\right) .
$$

\footnotetext{
${ }^{5} \mathrm{Au}$ cours de cette preuve, $\mathrm{TP}_{i}$ désignera la quantité d'où provient la contribution principale et $\mathrm{Err}_{i}$ un terme d'erreur.
} 
Estimation du terme d'erreur Err 1 . Découpons Err 1 de la façon suivante :

$$
\operatorname{Err}_{1}=2 \sum_{(m, N)=1} \sum_{1 \leqslant n \leqslant \frac{N Y \psi(N Y)}{m^{2}}} \cdots+2 \sum_{(m, N)=1} \sum_{n>\frac{N Y \psi(N Y)}{m^{2}}} \cdots:=\text { Error }+ \text { Err }_{3}
$$

pour toute fonction $\psi$ positive et tendant vers 0 en $+\infty$. Les estimations (4.2) et (4.3) assurent que

$$
\text { Error } \ll(N Y)^{1 / 4} \sum_{1 \leqslant m \leqslant \sqrt{N Y \psi(N Y)}} \frac{1}{m^{3 / 2}} \sum_{1 \leqslant n \leqslant \frac{N Y \psi(N Y)}{m^{2}}} \frac{\left|a_{n}\right|}{n^{\frac{3}{4}}} \exp \left(-4 \pi \frac{m}{\sqrt{N Y}} \sqrt{n}\right) .
$$

L'inégalité de Cauchy-Schwarz entraîne que le carré de la somme en $n$ est borné par

$$
\left(\sum_{1 \leqslant n \leqslant \frac{N Y \psi(N Y)}{m^{2}}} \frac{a_{n}^{2}}{n^{2}}\right)\left(\sum_{1 \leqslant n \leqslant \frac{N Y \psi(N Y)}{m^{2}}} \sqrt{n} \exp \left(-8 \pi \frac{m}{\sqrt{N Y}} \sqrt{n}\right)\right) .
$$

La première somme est estimée par $\mathcal{O}(\log (N Y \psi(N Y)))$ alors que la deuxième est trivialement inférieure à

$$
\int_{1}^{\frac{N Y \psi(N Y)}{m^{2}}} \sqrt{t} \exp \left(-8 \pi \frac{m}{\sqrt{N Y}} \sqrt{t}\right) \mathrm{d} t \ll\left(\frac{N Y \psi(N Y)}{m^{2}}\right)^{\frac{3}{2}} .
$$

Ainsi, on a prouvé que Error $\ll N Y(\psi(N Y))^{\frac{3}{4}}(\log (N Y \psi(N Y)))^{\frac{1}{2}}$. De la même manière,

$\operatorname{Err}_{3} \ll(N Y)^{1 / 4} \sum_{m \geqslant 1} \frac{1}{m^{3 / 2}}\left(\sum_{n>\frac{N Y \psi(N Y)}{m^{2}}} \frac{a_{n}^{2}}{n^{2+\varepsilon}}\right)^{\frac{1}{2}}\left(\sum_{n>\frac{N Y \psi(N Y)}{m^{2}}} n^{\frac{1}{2}+\varepsilon} \exp \left(-8 \pi \frac{m}{\sqrt{N Y}} \sqrt{n}\right)\right)^{\frac{1}{2}}$

pour tout $\varepsilon>0$. Une intégration par parties assure que

$$
\operatorname{Err}_{3} \ll(N Y)^{1+\varepsilon}(\psi(N Y))^{\frac{1}{2}+\varepsilon} \exp \left(-\frac{1}{2} \sqrt{\psi(N Y)}\right)
$$

et on choisit alors $\psi(x):=(\log x)^{a}$ avec $2 \varepsilon<a<\frac{2}{3}$ de sorte que Err $3=o$ (Error) et que Error $\ll(N Y)(\log (N Y))^{\frac{3 a}{4}+\frac{1}{2}}=o((N Y) \log (N Y))$.

Contribution du terme principal $\mathrm{TP}_{1}$. Déterminons le comportement asymptotique de $\mathrm{TP}_{1}$ en appliquant une méthode développée dans $\underline{\underline{\mathrm{w}}}$ :

- la condition $d$ sans facteurs carrés est supprimée en introduisant $\sum_{a^{2} \mid d} \mu(a)$ puis la somme est coupée selon la taille des diviseurs $a$ de $d(a \leqslant A$ et $a>A)$ sachant que l'on revient à des discriminants sans facteurs carrés dans le cas des grands diviseurs ;

- pour tout entier $m=m_{1} m_{2}^{2}$ avec $\left(m_{1} m_{2}, N\right)=1$ et $m_{1}$ sans facteurs carrés, remarquons que $\chi_{d}(m)=\chi_{d}\left(m_{1}\right)$ si $\left(m_{2}, d\right)=1$ (et 0 sinon) puis que le développement 
de Fourier du caractère $\chi .\left(m_{1}\right)$ en terme de caractères additifs de module $m_{1}$ s'écrit

$$
\chi_{d}\left(m_{1}\right)=\frac{\overline{\varepsilon_{m_{1}}}}{\sqrt{m_{1}}} \sum_{0 \leqslant|r|<\frac{m_{1}}{2}} \chi_{N r}\left(m_{1}\right) e\left(\frac{\bar{N} r d}{m_{1}}\right)
$$

où $\bar{N}$ est l'inverse de $N$ modulo $m_{1}$ et $\varepsilon_{m_{1}}$ est le signe de la somme de Gauss de $\chi .\left(m_{1}\right)$.

La contribution principale provient alors du terme $r=0$ pour lequel $\chi_{0}\left(m_{1}\right)$ vaut 0 si $m_{1}>1$ et 1 sinon. En résumé,

$$
\mathrm{TP}_{1}=\mathrm{TP}_{2}+\mathrm{Err}_{4}+\mathrm{Err}_{5}
$$

où

$$
\begin{aligned}
\mathrm{TP}_{2} & :=2 \sum_{n \leqslant 1} \frac{a_{n^{2}}}{n^{2}} \sum_{\substack{a \leqslant A \\
(a, 4 N)=1}} \mu(a) \sum_{\left(m_{2}, a N\right)=1} \frac{1}{m_{2}^{2}} \sum_{q \mid m_{2}} \mu(q) \sum_{\substack{q d \in \mathcal{D}^{\prime} \\
\left(d, m_{2}\right)=1}} V\left(\frac{4 \pi^{2} n^{2} m_{2}^{4}}{N a^{2}|d| q}\right) F\left(\frac{a^{2}|d| q}{Y}\right), \\
\mathrm{Err}_{5} & :=2 \sum_{n \leqslant 1} \frac{a_{n^{2}}}{n^{2}} \sum_{(b, 4 N)=1} \sum_{\substack{a \mid b \\
a>A}} \mu(a) \sum_{(m, N)=1} \frac{1}{m} \sum_{d \in \mathcal{D}} \chi_{b^{2} d}(m) V\left(\frac{4 \pi^{2} n^{2} m^{2}}{N b^{2}|d|}\right) F\left(\frac{b^{2}|d|}{Y}\right)
\end{aligned}
$$

et

$$
\begin{aligned}
\operatorname{Err}_{4}:=2 \sum_{n \leqslant 1} \frac{a_{n^{2}}}{n^{2}} \sum_{\substack{a \leqslant A \\
(a, 4 N)=1}} \mu(a) \sum_{\substack{m=m_{1} m_{2}^{2} \\
(m, a N)=1}} \frac{\mu^{2}\left(m_{1}\right)}{m} \sum_{q \mid m_{2}} \mu(q) \sum_{\substack{q d \in \mathcal{D}^{\prime} \\
\left(d, m_{2}\right)=1}} \\
\frac{\overline{\varepsilon_{m_{1}}}}{\sqrt{m_{1}}} \sum_{1 \leqslant|r|<\frac{m_{1}}{2}} \chi_{N r q}\left(m_{1}\right) e\left(\frac{\bar{N} r d}{m_{1}}\right) V\left(\frac{4 \pi^{2} n^{2} m^{2}}{N a^{2}|d| q}\right) F\left(\frac{a^{2}|d| q}{Y}\right)
\end{aligned}
$$

avec

$$
\mathcal{D}^{\prime}:=\left\{d \in \mathbb{Z}_{-}^{*}, d \equiv \nu^{2} \quad \bmod { }_{4} N,\left(\nu,{ }_{4} N\right)=1\right\} .
$$

Estimation du terme d'erreur Err. . Pour commencer, l'inégalité de Hölder implique que

$$
\operatorname{Er}_{5} \ll \sum_{n \ll(N Y)^{\frac{1}{2}}} \frac{\left|a_{n^{2}}\right|}{n^{2}} \sum_{\substack{b \geqslant 1 \\ a \mid b \\ a>A}}\left(\sum_{\substack{d \in \mathcal{D} \\|d| \ll \frac{Y}{b^{2}}}} 1\right)^{\frac{3}{4}}\left(\sum_{\substack{d \in \mathcal{D} \\|d| \ll \frac{Y}{b^{2}}}} \chi_{b^{2} d}(m)\left|\sum_{m \ll\left(\frac{N Y}{n^{2}}\right)^{\frac{1}{2}}} \frac{1}{m}\right|^{4}\right)^{\frac{1}{4}}
$$

d'où trivialement

$$
\operatorname{Err}_{5} \ll Y^{\frac{3}{4}} \sum_{n \ll(N Y)^{\frac{1}{2}}} \frac{\left|a_{n^{2}}\right|}{n^{2}} \sum_{\substack{b \geqslant 1 \\ a \mid b \\ a>A}} \frac{1}{b^{\frac{3}{2}}}\left(\sum_{\substack{d \in \mathcal{D} \\|d| \ll \frac{Y}{b^{2}}}} \chi_{b^{2} d}(m)\left|\sum_{m \ll\left(\frac{N Y}{n^{2}}\right)^{\frac{1}{2}}} \frac{1}{m}\right|^{2}\right)^{\frac{1}{2}} .
$$


L'inégalité du grand crible ([$[\mathrm{Bo}])$ pour les caractères réels assure alors que

$$
\operatorname{Err}_{5} \ll_{\varepsilon} \frac{Y^{\frac{5}{4}+\varepsilon}}{A^{\frac{3}{2}}}+\frac{N^{\frac{1}{4}+\varepsilon} Y^{1+\varepsilon}}{A^{\frac{1}{2}}}
$$

pour tout $\varepsilon>0$.

Estimation du terme d'erreur Err 4 . Posons $\Delta:=\inf \left(\frac{1}{2}, \frac{a^{2} q}{Y^{1-\varepsilon}}\right)$ pour tout nombre réel $\varepsilon>0$ et découpons $\mathrm{Err}_{4}$ selon que la sommation en $r$ est restreinte par

$$
\begin{gathered}
1 \leqslant|r|<\Delta m_{1} \\
\Delta m_{1} \leqslant|r|<\frac{m_{1}}{2} \rightsquigarrow \operatorname{Err}_{6}, \\
\operatorname{Err}_{7} .
\end{gathered}
$$

On estime Err 7 en bornant la somme sur les discriminants grâce au lemme 2 page 372 de $\underline{I w}$ puis trivialement la somme en $n$ et $m$ ce qui entraine que

$$
\operatorname{Err}_{7} \ll_{\varepsilon} \gamma(4 N) Y^{\varepsilon} N^{\frac{5}{4}+\varepsilon} \frac{\inf \left(A, Y^{\frac{1-\varepsilon}{2}}\right)}{Y^{\varepsilon-\frac{1}{4}}}+\gamma(4 N) Y^{\varepsilon} N^{\frac{5}{4}+\varepsilon} \frac{A^{3}}{Y^{\frac{3}{4}}}
$$

où $\gamma(4 N)$ est le cardinal de l'ensemble des classes d'équivalence de $\mathcal{D}^{\prime}$ modulo $4 N$. On estime Err $_{6}$ de façon triviale par

$$
\operatorname{Err}_{6} \ll_{\varepsilon} N^{\frac{3}{8}+\varepsilon} Y^{\frac{1}{2}+\varepsilon} A^{\frac{3}{2}} .
$$

Contribution du terme principal $\mathrm{TP}_{2}$. Intéressons-nous au terme principal $\mathrm{TP}_{2}$ et plus précisément à la somme sur les discriminants intervenant dans cette somme. Pour cela, on note $\mathcal{D}^{\prime}(4 N)$ l'ensemble des classes d'équivalence de $\mathcal{D}^{\prime}$ modulo $4 N$ et on se souvient que $\# \mathcal{D}^{\prime}(4 N)=\gamma(4 N)$. La formule de Poisson assure que

$$
\begin{aligned}
\sum_{q d \in \mathcal{D}^{\prime}} V\left(\frac{4 \pi^{2} n^{2} m_{2}^{4}}{N a^{2}|d| q}\right) F & \left(\frac{a^{2}|d| q}{Y}\right)=\frac{Y}{4 N a^{2} q} \sum_{\left[d_{0}\right] \in \mathcal{D}^{\prime}(4 N)} \sum_{\ell \in \mathbb{Z}} \\
& \times \int_{\mathbb{R}} V\left(\frac{4 \pi^{2} n^{2} m_{2}^{4}}{N Y\left|\frac{a^{2} q d_{0}}{Y}+t\right|}\right) F\left(\left|\frac{a^{2} q d_{0}}{Y}+t\right|\right) e\left(\frac{Y \ell}{4 N a^{2} q} t\right) \mathrm{d} t .
\end{aligned}
$$

On isole alors le terme $\ell=0$ et on effectue deux intégrations par parties pour chaque terme $\ell \neq 0$ afin de rendre absolument convergente la série en $\ell$ (il ne reste pas de termes entre crochets car $F$ est à support compact). On obtient alors

$$
\begin{array}{r}
\mathrm{TP}_{2}=\frac{\gamma(4 N) Y}{2 N} \int_{0}^{+\infty} F(t)\left(\sum_{n \geqslant 1} \frac{a_{n^{2}}}{n^{2}} \sum_{\substack{a \leqslant A \\
(a, 4 N)=1}} \frac{\mu(a)}{a^{2}} \sum_{\left(m_{2}, a N\right)=1} \frac{1}{m_{2}^{2}} \sum_{q \mid m_{2}} \frac{\mu(q)}{q} V\left(\frac{4 \pi^{2} n^{2} m_{2}^{4}}{N Y t}\right)\right) \mathrm{d} t \\
+\mathcal{O}\left(\frac{N^{\frac{5}{4}} \gamma(4 N) A^{3}}{Y^{\frac{3}{4}}}\right)
\end{array}
$$


Finalement,

$$
\mathrm{TP}_{2}=c_{N} Y \int_{0}^{+\infty} F(t) \mathcal{B}(N Y t) d t+\mathcal{O}\left(\frac{N^{\frac{1}{4}} \gamma(4 N) Y^{\frac{5}{4}}}{A}+\frac{N^{\frac{5}{4}} \gamma(4 N) A^{3}}{Y^{\frac{3}{4}}}\right)
$$

avec

$$
\mathcal{B}(X)=\sum_{n \geqslant 1} \frac{a_{n^{2}}}{n^{2}} \sum_{(m, N)=1} \frac{b_{m^{2}}}{m^{2}} V\left(\frac{4 \pi^{2} n^{2} m^{4}}{X}\right)
$$

et

$$
b_{m}=\prod_{\substack{p \in \mathcal{P} \\ p \mid m \\ p \neq 2}}\left(1+\frac{1}{p}\right)^{-1} .
$$

En revenant à la définition intégrale de la fonction $V$, on remarque que

$$
\mathcal{B}(X)=\frac{1}{2 i \pi} \int_{(3 / 4)} \Gamma(s)^{2} X^{-s} L(s+1) \mathrm{d} s
$$

et que le produit Eulérien intervenant dans la fonction $L$ est absolument convergeant sur $\Re(s)>\frac{3}{4}$ et y définit une fonction holomorphe. En décalant le contour jusqu'à $\left(-\frac{1}{4}+\varepsilon\right)$ pour tout $\varepsilon>0$, on ne croise qu'un pôle en $s=0$ ce qui prouve que

$$
\mathcal{B}(X)=-2(\gamma+\log (2 \pi)) L(1)+L^{\prime}(1)+L(1) \log (X)+\mathcal{O}_{\varepsilon}\left(\left(\frac{N}{X}\right)^{\frac{1}{4}+\varepsilon}\right) .
$$

Bilan et choix des paramètres. On a prouvé que

$$
S_{N}(Y)=\widetilde{\alpha_{N}} Y \log Y+\widetilde{\beta_{N}} Y+\mathcal{O}_{\varepsilon}\left(N Y(\log (N Y))^{\frac{1}{2}+\varepsilon}\right)+\mathrm{Err}
$$

où

$$
\operatorname{Err} \ll_{\varepsilon}(N Y)^{\varepsilon}\left(\frac{Y^{\frac{5}{4}}}{A^{\frac{3}{2}}}+\frac{N^{\frac{1}{4}} Y}{A^{\frac{1}{2}}}+\frac{N^{\frac{9}{4}} \inf \left(A, Y^{\frac{1-\varepsilon}{2}}\right)}{Y^{\varepsilon-\frac{1}{4}}}+\frac{N^{\frac{9}{4}} A^{3}}{Y^{\frac{3}{4}}}+N^{\frac{3}{8}} Y^{\frac{1}{2}+\varepsilon} A^{\frac{3}{2}}+\frac{N^{\frac{5}{4}} Y^{\frac{5}{4}}}{A}\right)
$$

et on choisit alors $A:=N^{\frac{1}{2}} Y^{\frac{3}{10}-\frac{2 \varepsilon}{5}}$ ce qui achève la preuve.

Appliquons finalement la formule de Gross-Zagier (2.2) pour obtenir une estimation asymptotique de la hauteur en moyenne des points de Heegner de la même forme que celle que l'on avait obtenue pour les traces.

Corollaire 4.2. Si E est une courbe elliptique rationnelle de conducteur $N$ sans facteurs carrés et de rang analytique quelconque alors

$$
\sum_{\substack{d \in \mathcal{D} \\|d| \leqslant Y}} \hat{h}_{\mathbb{H}_{d}}\left(\mathrm{P}_{d}\right)=C_{\mathrm{P}} Y^{\frac{3}{2}} \log Y+C_{\mathrm{P}}^{\prime} Y^{\frac{3}{2}}+\frac{1}{3 \Omega_{E, N}} \sqrt{Y} \text { Error }+\mathcal{O}_{\varepsilon}\left(N^{\frac{15}{4}+\varepsilon} Y^{\frac{29}{20}+\varepsilon}\right)
$$


ò̀

$$
\frac{1}{3 \Omega_{E, N}} \sqrt{Y} \text { Error }=\mathcal{O}_{\varepsilon}\left(N Y^{\frac{3}{2}}(\log (N Y))^{\frac{1}{2}+\varepsilon}\right)
$$

pour tout $\varepsilon>0$ et où $C_{\mathrm{P}}$ est la constante définie par

$$
C_{\mathrm{P}}:=\frac{2}{\pi} c_{N} \mathcal{Q}(N) \frac{L\left(S_{\left.y m^{2} E, 2\right)}\right.}{\pi \Omega_{E, N}} \prod_{\substack{p \in \mathcal{P} \\ p \mid N}}\left(1-\frac{1}{p^{2}}\right)^{-1}
$$

avec

$$
\mathcal{Q}(N):=\prod_{\substack{p \in \mathcal{P} \\ p \nmid 2 N}}\left(1+\left(1+\frac{1}{p}\right)^{-1}\left(p^{2}-1\right)^{-1}\right) \times \begin{cases}\frac{4}{3} & \text { si } N \text { est impair } \\ 1 & \text { sinon }\end{cases}
$$

et

$$
C_{\mathrm{P}}^{\prime}:=C_{\mathrm{P}}\left(\log \left(\frac{N}{4 \pi^{2}}\right)-\frac{2}{3}-2 \gamma\right)+\frac{c_{N}}{3 \Omega_{E, N}} \widetilde{L}^{\prime}(1) .
$$

Remarque 4.2. En accord avec la remarque 4.1 on peut conjecturer que

$$
\frac{1}{3 \Omega_{E, N}} \sqrt{Y} \text { Error }=o_{\varepsilon}\left(N Y^{\frac{3}{2}}\right)
$$

et le corollaire précédent semble alors nous munir d'un développement asymptotique à deux termes de la hauteur en moyenne des points de Heegner.

Remarque 4.3. En remplaçant $c_{N}$ et $L\left(\mathrm{Sym}^{2} E, 2\right)$ par leur expression on peut réécrire

$$
C_{\mathrm{P}}=\left(\left(\frac{8}{\pi^{3} c_{E}(N)^{2}}\right) \mathcal{Q}(N) \prod_{\substack{p \in \mathcal{P} \\ p \mid N}}\left(1-\frac{1}{p^{2}}\right)^{-2}\right) \frac{\gamma(4 N)}{N^{2}} \operatorname{deg}\left(\phi_{N, E}\right) .
$$

Ainsi, contrairement à la constante $C_{\operatorname{Tr}}$ intervenant lorsque l'on considère les traces, à conducteur fixé $C_{\mathrm{P}}$ ne dépend que du degré de la paramétrisation modulaire, puisque le produit $\mathcal{Q}(N)$ ne dépend que de $N$. Par contre, lorsque l'on varie le conducteur, il n'y a plus une dépendance directe sur le degré. Il est clair que $\mathcal{Q}(N) \geqslant 1$; d'autre part

$$
\mathcal{Q}(N)<\frac{4}{3} \prod_{p}\left(1+\frac{1}{p^{2}}\right)<\frac{4}{3} \zeta(2) .
$$

Le produit Eulerien $\mathcal{Q}(N)$ est donc compris entre 1 et 2 , et il joue un relativement faible rôle dans l'expression de $C_{\mathrm{P}}$. On a ainsi

$$
1 \leqslant \prod_{p \mid N}\left(1-\frac{1}{p^{2}}\right)^{-2} \mathcal{Q}(N) \leqslant \frac{4}{3} \zeta(2)^{3}<6 .
$$

Le terme principal, du moins si l'on s'intéresse à des valeurs asymptotiques du conducteur ou du degré de la paramétrisation modulaire, est donc $\frac{\gamma(4 N)}{N^{2}} \operatorname{deg}\left(\phi_{N, E}\right)$. Selon la conjecture du degré (cf. [Mu] et [De] page 35) qui est équivalente à une des formes de 
la conjecture abc, on aurait $\operatorname{deg}\left(\Phi_{N, E}\right) \ll_{\varepsilon} N^{2+\epsilon}$ pour tout $\epsilon>0$. Comme $\gamma(4 N) \ll N$, cela donne une borne supérieure sur la croissance des hauteurs des points $\mathrm{P}_{d}$ lorsque $N$ tend vers $+\infty$ avec $Y$. On sait d'autre part qu'il existe des familles de courbes de $j$-invariant borné ([De] page 50) pour lesquelles $\operatorname{deg}\left(\Phi_{N, E}\right) \gg N^{\frac{7}{6}} \log N$ ce qui donne une borne inférieure sur la vitesse de croissance des hauteurs lorsque $N$ tend vers $+\infty$ avec $Y$.

Remarque 4.4. Remarquons finalement que, même à conducteur fixé, la constante $C_{P}^{\prime}$ dépend de la courbe elliptique $E$ et pas seulement du degré de la paramétrisation modulaire de $E$. Par contre, il ne semble pas être possible d'obtenir une estimation satisfaisante de la taille de $C_{P}^{\prime}$ par rapport au niveau.

\section{Analyse Des Résultats théoriques et numériques}

Après avoir donné quelques valeurs numériques des constantes en jeu, nous donnons des résultats expérimentaux illustrant les formules théoriques.

\subsection{Quelques valeurs numériques.}

5.1.1. Valeurs numériques de $C_{T r}$ et de $C_{P}$. Le tableau 1 regroupe les valeurs des trois constantes $C_{\mathrm{Tr}}^{(0)}, C_{\mathrm{Tr}}^{(1)}$ et $C_{\mathrm{P}}$ régissant le comportement en moyenne des hauteurs des points de Heegner et de leurs traces (selon les corollaires 3.2, 3.4 et 4.2) pour toutes les courbes elliptiques de conducteur sans facteurs carrés et inférieur à 100. Les valeurs de $C_{\operatorname{Tr}}$ et de $C_{\mathrm{P}}$ ont été multipliées par $10^{3}$ pour une meilleure lisibilité. Le rapport entre la constante gouvernant le comportement des hauteurs des points et celle donnant celui des traces donné dans la dernière colonne n'a que peu de sens dans le cas d'une courbe elliptique de rang 1 car les points y sont asymptotiquement «plus gros» que les traces d'un facteur $\log Y$ selon les corollaires 3.4 et 4.2. Il est intéressant de voir que cette constante prend à la fois des valeurs plus grandes («les points sont plus gros») et plus petites («les traces sont plus grosses») que 1 .

Remarque 5.1. Si l'on poursuit le calcul sur les 200 premières courbes elliptiques de conducteur sans facteurs carrés alors on obtient un rapport moyen de 1.5 environ et ce rapport tend à décroître. Il n'y a donc pas de raison a priori de croire qu'il soit plus souvent plus grand ou petit que 1 .

5.1.2. Étude plus fine du rapport $C_{P} / C_{T r}^{(0)}$. Pour étudier le rapport

$$
\frac{C_{\mathrm{P}}}{C_{\mathrm{Tr}}^{(0)}}=\frac{\mathcal{Q}(N)}{\mathcal{P}(1)} L(E, 1)^{-1} \prod_{p \mid N}\left(1-\frac{a_{p}}{p}\right)\left(1-\frac{a_{p^{2}}}{p^{2}}\right)^{-1},
$$




\begin{tabular}{|c|c|c|c|c|c|c|c|c|c|}
\hline Courbe & Rang & $C_{\operatorname{Tr}} \times 10^{3}$ & $C_{\mathrm{P}} \times 10^{3}$ & $C_{\mathrm{P}} / C_{\operatorname{Tr}}$ & Courbe & Rang & $C_{\operatorname{Tr}}$ & $C_{\mathrm{P}}$ & $C_{\mathrm{P}} / C_{\operatorname{Tr}}$ \\
\hline $11-1$ & 0 & 3.33 & 17.0 & 5.11 & $58-1$ & 1 & 1.14 & 6.80 & 5.91 \\
\hline $14-1$ & 0 & 1.07 & 6.39 & 5.92 & $58-2$ & 0 & 9.47 & 6.80 & 0.718 \\
\hline $15-1$ & 0 & 0.904 & 4.40 & 4.87 & $61-1$ & 1 & 1.97 & 6.58 & 3.33 \\
\hline $17-1$ & 0 & 3.21 & 11.3 & 3.52 & $62-1$ & 0 & 4.85 & 3.18 & 0.657 \\
\hline $19-1$ & 0 & 3.10 & 10.2 & 3.29 & $65-1$ & 1 & 0.593 & 2.44 & 4.12 \\
\hline $21-1$ & 0 & 1.21 & 3.28 & 2.71 & $66-1$ & 0 & 1.01 & 2.18 & 2.15 \\
\hline $26-1$ & 0 & 1.80 & 7.29 & 4.03 & $66-2$ & 0 & 2.01 & 2.18 & 1.08 \\
\hline $26-2$ & 0 & 5.00 & 7.29 & 1.45 & $66-3$ & 0 & 25.2 & 10.9 & 0.433 \\
\hline $30-1$ & 0 & 0.621 & 2.20 & 3.54 & $67-1$ & 0 & 12.4 & 15.0 & 1.20 \\
\hline $33-1$ & 0 & 2.48 & 6.56 & 2.64 & $69-1$ & 0 & 2.40 & 2.18 & 0.909 \\
\hline $34-1$ & 0 & 5.11 & 5.67 & 1.10 & $70-1$ & 0 & 2.49 & 2.14 & 0.861 \\
\hline $35-1$ & 0 & 1.95 & 4.29 & 2.19 & $73-1$ & 0 & 6.90 & 8.27 & 1.19 \\
\hline $37-1$ & 1 & 1.86 & 10.7 & 5.75 & $77-1$ & 1 & 1.24 & 4.26 & 3.42 \\
\hline $37-2$ & 0 & 5.64 & 10.7 & 1.90 & $77-2$ & 0 & 15.4 & 21.3 & 1.37 \\
\hline $38-1$ & 0 & 4.70 & 15.3 & 3.25 & $77-3$ & 0 & 5.20 & 6.39 & 1.23 \\
\hline $38-2$ & 0 & 5.09 & 5.10 & 1.00 & $78-1$ & 0 & 4.47 & 18.7 & 4.18 \\
\hline $39-1$ & 0 & 1.54 & 3.75 & 2.43 & $79-1$ & 1 & 1.97 & 5.10 & 2.58 \\
\hline $42-1$ & 0 & 2.30 & 3.28 & 1.42 & $82-1$ & 1 & 1.15 & 4.85 & 4.19 \\
\hline $43-1$ & 1 & 1.90 & 9.27 & 4.87 & $83-1$ & 1 & 1.93 & 4.85 & 2.51 \\
\hline $46-1$ & 0 & 3.16 & 10.6 & 3.36 & $85-1$ & 0 & 3.00 & 3.80 & 1.26 \\
\hline $51-1$ & 0 & 2.12 & 2.91 & 1.37 & $89-1$ & 1 & 1.90 & 4.53 & 2.37 \\
\hline $53-1$ & 1 & 1.87 & 7.55 & 4.02 & $89-2$ & 0 & 10.5 & 11.3 & 1.07 \\
\hline $55-1$ & 0 & 2.33 & 2.85 & 1.22 & $91-1$ & 1 & 1.09 & 3.65 & 3.34 \\
\hline $57-1$ & 1 & 0.923 & 5.24 & 5.68 & $91-2$ & 1 & 2.03 & 3.65 & 1.79 \\
\hline $57-2$ & 0 & 3.83 & 3.93 & 1.02 & $94-1$ & 0 & 4.11 & 2.12 & 0.516 \\
\hline $57-3$ & 0 & 8.24 & 15.7 & 1.90 & & & & & \\
\hline
\end{tabular}

TAB. 1 - Valeurs numériques des constantes $C_{\mathrm{Tr}}^{(0)}, C_{\operatorname{Tr}}^{(1)}$ et $C_{\mathrm{P}}$.

on néglige le rôle de $\frac{\mathcal{Q}(N)}{\mathcal{P}(1)}$, qui est de toute façon borné. Ainsi, la taille de

$$
\gamma_{E}=L(E, 1)^{-1} \prod_{p \mid N}\left(1+\frac{a_{p}}{p}\right)^{-1}
$$

par rapport à 1 reflète essentiellement le signe du terme

$$
\sum_{\sigma \in G_{d} \backslash\{I d\}}<P, P^{\sigma}>_{\mathbb{H}_{d}}
$$


Plus $\gamma_{E}$ sera petit, plus ce produit scalaire sera grand, et, de manière imagée, on pourrait dire que les points de Heegner sont essentiellement resserés autour d'une même direction ; alors que si $\gamma_{E}$ est grand devant 1, cette somme est négative et les points sont éclatés dans l'espace à $h_{d}$ dimensions. On s'attend donc, par exemple, à ce que la hauteur des traces (en moyenne) soit supérieure à celle des points sur la courbe $58 \mathrm{~B}\left(\gamma_{E}=0.67\right)$, ce qui est illustré par la figure 5 . Par contre, dans le cas de la courbe 37B $\left(\gamma_{E}=1.34\right)$, les points sont plus gros (figure [6).

Somme des hauteurs

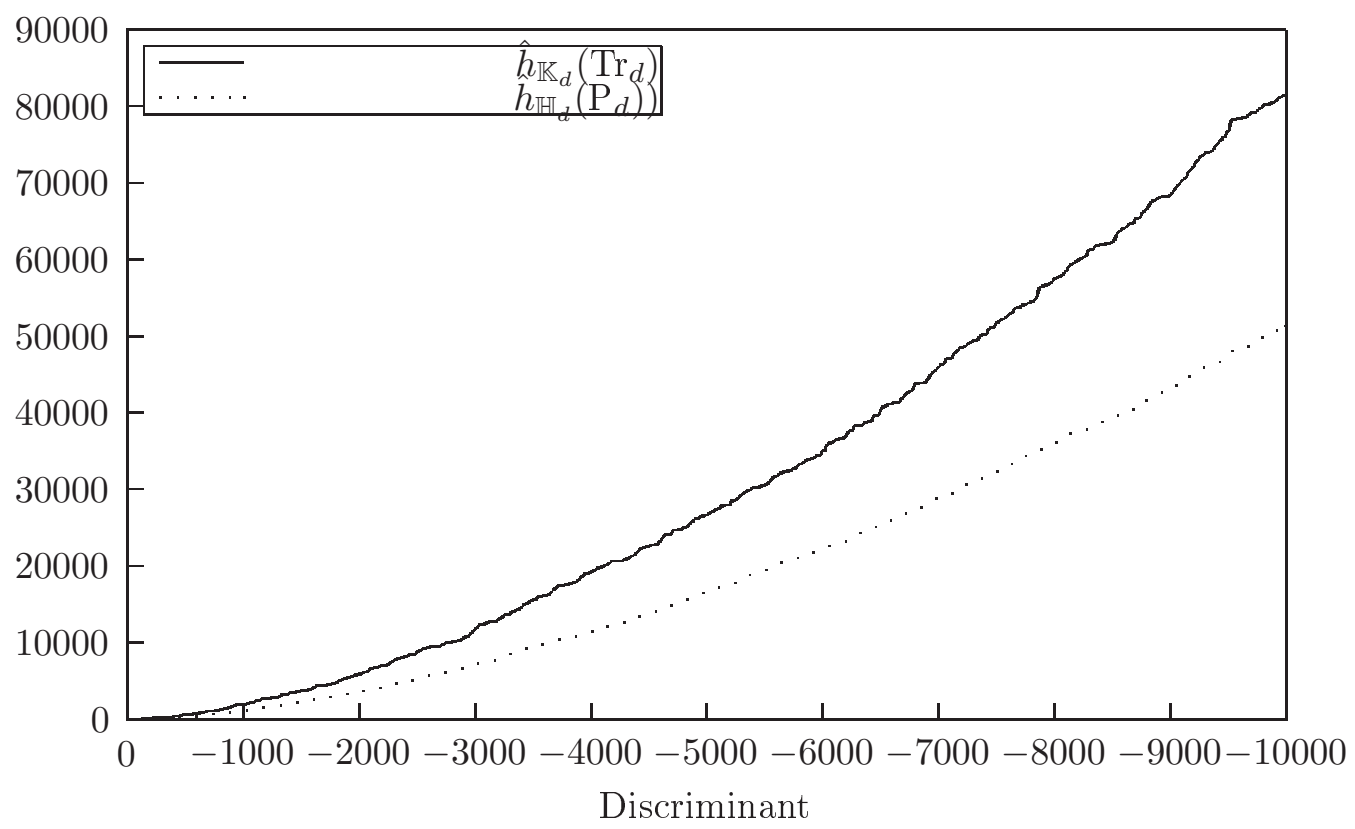

Fig. 5 - Somme des hauteurs des traces et des points sur la courbe 58B.

5.2. Résultats expérimentaux. Nous avons effectué de nombreux calculs de points de Heegner et de leurs hauteurs sur différentes courbes, à l'aide des logiciels Magma et Pari. Magma a permis de calculer les points, ou les traces, eux-mêmes (suivant la méthode de calcul exposée dans $[\overline{\mathrm{DaGr}}$ ), alors que Pari s'est avéré plus rapide pour le calcul direct de la série $L$ intervenant dans la formule de Gross-Zagier. On s'est concentré sur des courbes de petits conducteurs $(N<200)$, car les algorithmes ont une complexité en $\mathcal{O}\left(N^{2}\right)$. Nous présentons ici certains des résultats obtenus, pour illustrer notre théorème. 
Somme des hauteurs

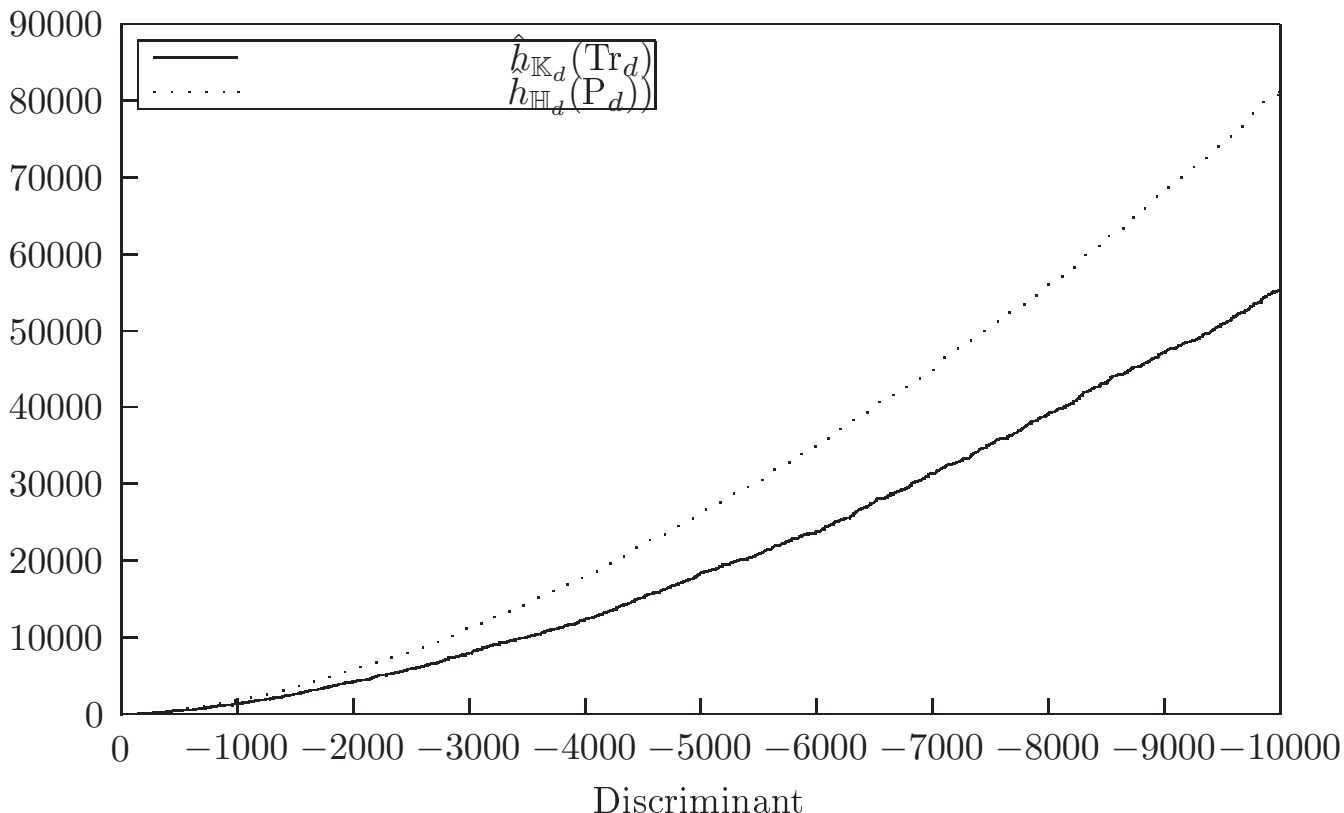

Fig. 6 - Somme des hauteurs des traces et des points sur la courbe 37B.

5.2.1. Comparaison entre les valeurs expérimentale et théorique de $C_{P}$. Nous commençons par comparer les valeurs expérimentales de $C_{\mathrm{P}}$ à la valeur théorique donnée dans la section précédente. Ainsi, pour chaque courbe de conducteur sans facteurs carrés plus petit que 100, on a représenté le rapport entre la valeur expérimentale

$$
C_{\mathrm{P}}^{\exp }(Y):=\frac{1}{Y^{3 / 2} \log Y} \sum_{\substack{d \in \mathcal{D} \\|d| \leqslant Y}} \hat{h}_{\mathbb{H}_{d}}\left(\mathrm{P}_{d}\right)
$$

pour quelques valeurs de $Y$ et la valeur théorique dans le tableau 2 Ainsi, même pour des discriminants assez grands $\left(2 \cdot 10^{4}\right)$, la constante expérimentale est souvent de l'ordre de $75 \%$ de la constante théorique. On a représenté plusieurs valeurs de $Y$ pour bien montrer que ce rapport augmente toutefois, mais très lentement.

5.2.2. Étude plus fine des courbes $37 \mathrm{~A}$ et $37 \mathrm{~B}$. Nous allons étudier plus en profondeur les courbes $37 \mathrm{~A}$ et $37 \mathrm{~B}$. Ces deux courbes sont intéressantes pour plusieurs raisons : elles ont même degré et même conducteur, donc devraient avoir même $C_{\mathrm{P}}$. La courbe 37B est de rang 0 alors que la 37A est la courbe de rang 1 de plus petit conducteur. 


\begin{tabular}{|c|c|c|c|}
\hline Courbe & 6000 & 13000 & 20000 \\
\hline $11-1$ & 0.716 & 0.738 & 0.750 \\
$14-1$ & 0.707 & 0.736 & 0.742 \\
$15-1$ & 0.703 & 0.723 & 0.740 \\
$17-1$ & 0.735 & 0.753 & 0.764 \\
$19-1$ & 0.718 & 0.737 & 0.748 \\
$21-1$ & 0.700 & 0.731 & 0.742 \\
$26-1$ & 0.714 & 0.730 & 0.744 \\
$26-2$ & 0.682 & 0.702 & 0.717 \\
$30-1$ & 0.687 & 0.703 & 0.722 \\
$33-1$ & 0.697 & 0.725 & 0.736 \\
$34-1$ & 0.707 & 0.724 & 0.735 \\
$35-1$ & 0.749 & 0.764 & 0.774 \\
$37-1$ & 0.661 & 0.689 & 0.704 \\
$37-2$ & 0.805 & 0.820 & 0.830 \\
$38-1$ & 0.760 & 0.775 & 0.784 \\
$38-2$ & 0.702 & 0.722 & 0.733 \\
$39-1$ & 0.713 & 0.720 & 0.738 \\
\hline
\end{tabular}

\begin{tabular}{|c|c|c|c|}
\hline Courbe & 6000 & 13000 & 20000 \\
\hline $42-1$ & 0.683 & 0.722 & 0.735 \\
$43-1$ & 0.678 & 0.699 & 0.714 \\
$46-1$ & 0.699 & 0.720 & 0.729 \\
$51-1$ & 0.711 & 0.719 & 0.737 \\
$53-1$ & 0.690 & 0.716 & 0.725 \\
$55-1$ & 0.818 & 0.821 & 0.825 \\
$57-1$ & 0.680 & 0.695 & 0.705 \\
$57-2$ & 0.774 & 0.780 & 0.785 \\
$57-3$ & 0.727 & 0.738 & 0.745 \\
$58-1$ & 0.663 & 0.687 & 0.703 \\
$58-2$ & 0.808 & 0.818 & 0.828 \\
$61-1$ & 0.735 & 0.752 & 0.770 \\
$62-1$ & 0.832 & 0.835 & 0.847 \\
$65-1$ & 0.752 & 0.756 & 0.766 \\
$66-1$ & 0.752 & 0.773 & 0.785 \\
$66-2$ & 0.693 & 0.719 & 0.734 \\
$66-3$ & 0.671 & 0.699 & 0.714 \\
\hline
\end{tabular}

\begin{tabular}{|c|c|c|c|}
\hline Courbe & 6000 & 13000 & 20000 \\
\hline $67-1$ & 0.725 & 0.753 & 0.763 \\
$69-1$ & 0.795 & 0.797 & 0.807 \\
$70-1$ & 0.762 & 0.774 & 0.785 \\
$73-1$ & 0.803 & 0.822 & 0.834 \\
$77-1$ & 0.709 & 0.738 & 0.752 \\
$77-2$ & 0.767 & 0.791 & 0.802 \\
$77-3$ & 0.770 & 0.795 & 0.806 \\
$78-1$ & 0.722 & 0.722 & 0.742 \\
$79-1$ & 0.794 & 0.823 & 0.827 \\
$82-1$ & 0.731 & 0.754 & 0.763 \\
$83-1$ & 0.786 & 0.799 & 0.811 \\
$85-1$ & 0.808 & 0.820 & 0.826 \\
$89-1$ & 0.823 & 0.838 & 0.844 \\
$89-2$ & 0.800 & 0.817 & 0.824 \\
$91-1$ & 0.738 & 0.748 & 0.754 \\
$91-2$ & 0.766 & 0.772 & 0.778 \\
$94-1$ & 0.996 & 0.980 & 0.974 \\
\hline
\end{tabular}

TAB. 2 - Rapport entre valeur expérimentale $C_{\mathrm{P}}^{\exp }(Y)$ et valeur théorique $C_{\mathrm{P}}$.

$\sum_{\substack{d \in \mathcal{D} \\|d| \leqslant Y}} \hat{h}_{\mathbb{H}_{d}}\left(\mathrm{P}_{d}\right)$

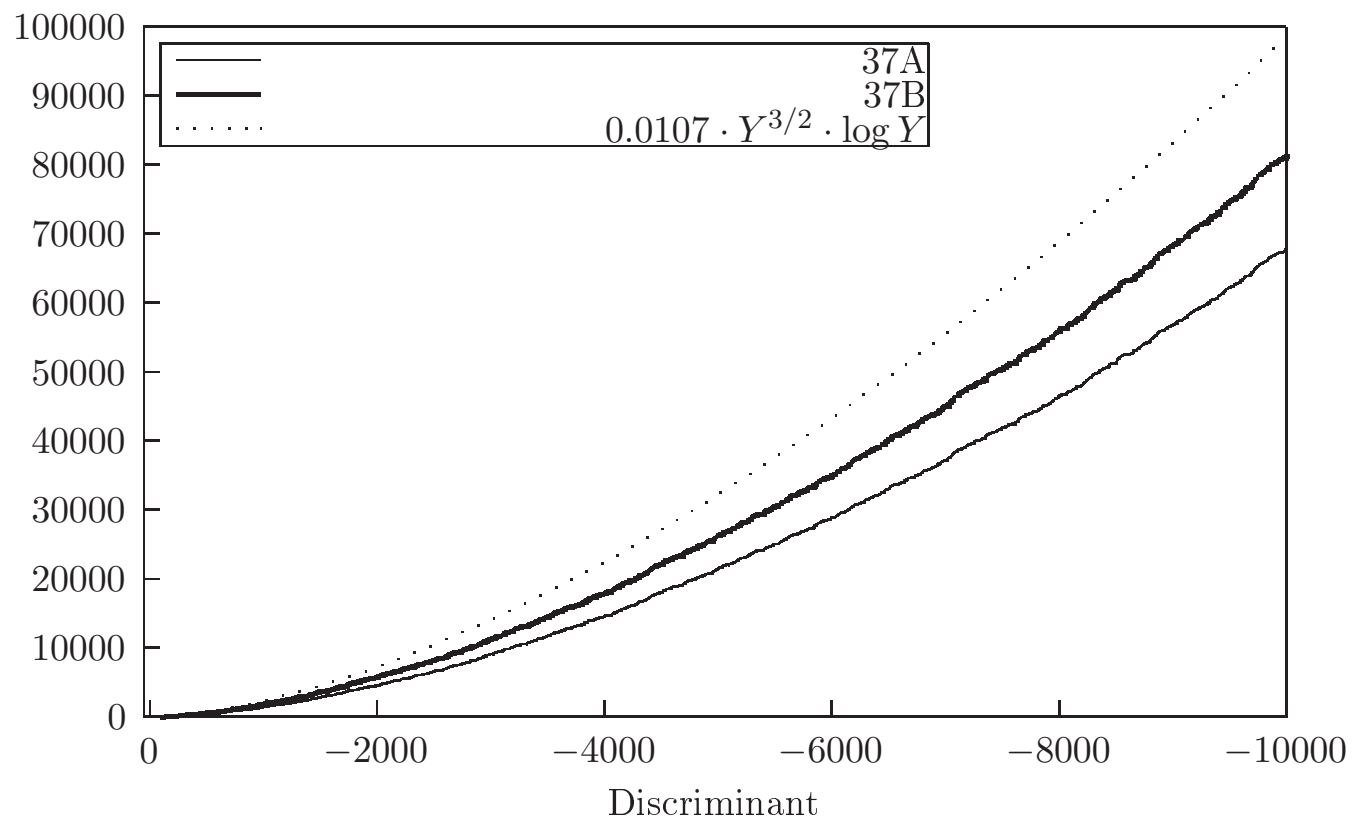

Fig. 7 - Hauteur des points en moyenne sur les courbes 37A et 37B. 
La figure 7 représente les sommes des hauteurs des points sur les courbes $37 \mathrm{~A}$ et $37 \mathrm{~B}$ comparées à la valeur théorique donnée par le corollaire 4.2. Contrairement à ce que l'on avait pour les hauteurs des traces, ici les courbes ne se supperposent pas du tout, ce qui était prévisible étant donné le tableau ci-dessus.

On a en particulier l'impression que la courbe 37A est nettement en-dessous de la 37B sans paraître la rejoindre alors que le corollaire 4.2 affirme que les hauteurs des points sur ces courbes elliptiques devraient être les mêmes en moyenne. Cependant, une analyse plus fine de la différence entre ces deux courbes montre qu'elle semble être en $Y^{3 / 2}$ et donc que les deux courbes semblent se rapprocher à une vitesse de $1 / \log Y$ de la courbe théorique d'équation $Y \mapsto 0.0107 Y^{\frac{3}{2}} \log Y$ ce qu'il est malheureusement difficile d'observer dans l'échelle de discriminants représentée. Autrement dit, on devine numériquement sur les courbes $37 \mathrm{~A}$ et $37 \mathrm{~B}$ que

$$
\sum_{\substack{d \in \mathcal{D} \\|d| \leqslant Y}} \hat{h}_{\mathbb{H}_{d}}\left(\mathrm{P}_{d}\right)=C_{\mathrm{P}} Y^{\frac{3}{2}} \log Y\left(1+\mathcal{O}_{N, E}\left(\frac{1}{\log Y}\right)\right) .
$$

Selon le corollaire 4.2 et la preuve du théorème 4.1 on a

$$
\begin{aligned}
\sum_{\substack{d \in \mathcal{D} \\
|d| \leqslant Y}} \hat{h}_{\mathbb{H}_{d}}\left(\mathrm{P}_{d}\right) & =C_{\mathrm{P}} Y^{\frac{3}{2}} \log Y+C_{\mathrm{P}}^{\prime} Y^{\frac{3}{2}}+\frac{1}{3 \Omega_{E, N}} Y^{\frac{1}{2}} \text { Error }+\mathcal{O}_{N, \varepsilon}\left(Y^{\frac{29}{20}+\varepsilon}\right), \\
& =C_{\mathrm{P}} Y^{\frac{3}{2}} \log Y\left(1+\frac{C_{\mathrm{P}}^{\prime}}{C_{\mathrm{P}}} \frac{1}{\log Y}+\frac{1}{3 \Omega_{E, N} C_{\mathrm{P}}} \frac{\text { Error }}{Y \log Y}+\mathcal{O}_{N, \varepsilon}\left(Y^{-\frac{1}{20}+\varepsilon}\right)\right)
\end{aligned}
$$

où Error est défini en (4.4) et pour tout $\varepsilon>0$. L'analyse numérique suggère donc que le terme $C_{\mathrm{P}} Y^{3 / 2}$ dans le développement du corollaire 4.2 est non nul et même de l'ordre du terme principal pour des petits discriminants. Ceci suggère également que

$$
\text { Error }=o_{\varepsilon}(N Y) \text {. }
$$

Prouver cela nécessite de pouvoir estimer les moyennes mentionnées dans la remarque 4.1. Donnons une autre justification numérique de nos intuitions. Posons

$$
\delta(Y):=\frac{1}{Y^{3 / 2}} \sum_{\substack{d \in \mathcal{D} \\|d| \leqslant Y}}\left(\hat{h}_{\mathbb{H}_{d}, 37 \mathrm{~B}}\left(\mathrm{P}_{d}\right)-\hat{h}_{\mathbb{H}_{d}, 37 \mathrm{~A}}\left(\mathrm{P}_{d}\right)\right) .
$$

Le tableau 3 donne la valeur de $\delta(Y)$ pour plusieurs valeurs de $Y$. Ainsi, $\delta(Y)$ décroît

\begin{tabular}{|c|c|c|c|c|c|}
\hline$Y$ & $2 \cdot 10^{4}$ & $4 \cdot 10^{4}$ & $6 \cdot 10^{4}$ & $8 \cdot 10^{4}$ & $10 \cdot 10^{4}$ \\
\hline$\delta(Y)$ & 0.01337 & 0.01329 & 0.01328 & 0.01326 & 0.01324 \\
\hline
\end{tabular}

TAB. 3 - Valeurs numériques de $\delta(Y)$.

très légèrement avec $Y$ et semble se stabiliser. On devine alors que

$$
\delta(Y)=D_{E}+o_{N, E}(1)
$$


pour une constante $D_{E}$. Or, le corollaire 4.2 affirme que

$$
\delta(Y)=\left(C_{\mathrm{P}, 37 \mathrm{~B}}-C_{\mathrm{P}, 37 \mathrm{~A}}\right)+\frac{1}{3 Y}\left(\frac{\text { Error }_{37 \mathrm{~B}}}{\Omega_{37 \mathrm{~B}, 37}}-\frac{\text { Error }_{37 \mathrm{~A}}}{\Omega_{37 \mathrm{~A}, 37}}\right)+\mathcal{O}_{N, \varepsilon}\left(Y^{-\frac{1}{20}+\varepsilon}\right)
$$

pour tout $\varepsilon>0$ ce qui confirme (5.1). En outre, il ne semble pas y avoir de compensation entre $C_{\mathrm{P}}^{\prime} Y^{\frac{3}{2}}$ et $C_{\mathrm{P}}^{\prime \prime} Y^{\frac{1}{2}} \operatorname{Err}_{1}$ car sinon $\delta(Y)$ tendrait plus vite vers 0 .

\section{RÉFÉRENCES}

[AbUl] Abbes A., Ullmo E. : À propos de la conjecture de Manin pour les courbes elliptiques modulaires, Compositio Math. 103 :3 (1996), 269-286.

[Bo] Bombieri E. : Le grand crible dans la Théorie Analytique des Nombres, Astérisque 18 (1973).

[Cr] Cremona J.E. : Elliptic Curve Data, disponible àhttp://www.maths.nott.ac.uk/personal/jec/ftp/data/INDEX.html

[Da] Darmon H. : Rational points on modular elliptic curves, CBMS vol. 101 (2004).

[DaGr] Darmon H., Green P. : Elliptic curves and class fields of real quadratic fields : algorithms and evidence, Exp. Math. 11(2002), 37-55.

[De] Delaunay C. : Formes modulaires et invariants de courbes elliptiques définies sur $\mathbb{Q}$, thèse de doctorat, Université Bordeaux I (2002), disponible à http://igd.univ-lyon1.fr/ delaunay/

[Ed] Edixhoven B. : On the Manin constants of modular elliptic curves, in Arithmetic Algebraic Geometry (Texel, 1989), edited by G. van der Geer, F. Oort, and J. Steenbrink, 25-39, Progr. Math. 89. Boston, MA : Birkhäuser Boston, 1991.

[Gr] Gross B.H. : Heegner Points on $X_{0}(N)$, Modular Forms, ed. R.A. Rankin, Halsted Press (1984), 87-105.

[GrZa] Gross B.H., Zagier D., Derivatives of L-series and the height of Heegner points, Invent. Math. 84(1986), 225-320.

[Iw] Iwaniec H., On the order of vanishing of modular L-functions at the critical point, J. Théor. Nombres Bordeaux 6(1990), 365-375.

[IwKo] Iwaniec H., Kowalski E. : Analytic number theory, Providence R.I., Colloquium publications (American Mathematical Society) (2004).

[Ma] Manin J., Parabolic points and zeta functions of modular curves, Izv. Akad. Nauk SSSR Ser. Mat. 36 (1972), 19-66.

[Mu] Murty M.R., Bounds for congruence primes, Proc. Sympos. Pure Math. 66(1999), 177-192.

[TaWi] Taylor R., Wiles A. :Ring-theoretic properties of certain Hecke algebras, Ann. of Math. (2) 141 $553-572$.

[Sh] Shimura G., On the holomorphy of a certain Dirichlet series, Proc. London Math. Soc. 31(1975), 79-98.

[Si] Silverman J.H., The arithmetic of elliptic curves, Graduate Texts in Mathematics 106, Springer-Verlag, Berlin, 1986.

[Wa] Watkins M., Computing the modular degree of an elliptic curve, Experiment. Math. 11(2002).

[Wi] Wiles A. : Modular elliptic curves and Fermat's last theorem, Ann. of Math. (2) 141, 443-551.

\section{G. Ricotta}

Université de Montréal, Département de Mathématiques et de Statistique, CP 6128 succ 
Centre-Ville, Montréal QC H3C 3J7, Canada; ricotta@dms.umontreal.ca T. Vidick

École Normale Supérieure, 45 rue d’Ulm, 75005 Paris, France; thomas.vidick@ens.fr 